\title{
SPECTRAL ASYMPTOTICS FOR RESOLVENT DIFFERENCES OF ELLIPTIC OPERATORS WITH $\delta$ AND $\delta^{\prime}$-INTERACTIONS ON HYPERSURFACES
}

\author{
JUSSI BEHRNDT, GERD GRUBB, MATTHIAS LANGER, \\ AND VLADIMIR LOTOREICHIK
}

\begin{abstract}
We consider self-adjoint realizations of a second-order elliptic differential expression on $\mathbb{R}^{n}$ with singular interactions of $\delta$ and $\delta^{\prime}$-type supported on a compact closed smooth hypersurface in $\mathbb{R}^{n}$. In our main results we prove spectral asymptotics formulae with refined remainder estimates for the singular values of the resolvent difference between the standard self-adjoint realizations and the operators with a $\delta$ and $\delta^{\prime}$-interaction, respectively. Our technique makes use of general pseudodifferential methods, classical results on spectral asymptotics of $\psi$ do's on closed manifolds and Krein-type resolvent formulae.
\end{abstract}

\section{INTRODUCTION}

In this paper we study self-adjoint operator realizations of the formally symmetric, uniformly strongly elliptic differential expression

$$
(\mathcal{A} u)(x):=-\sum_{j, k=1}^{n} \partial_{j}\left(a_{j k}(x) \partial_{k} u\right)(x)+a(x) u(x), \quad x \in \mathbb{R}^{n},
$$

with singular interactions of $\delta$ and $\delta^{\prime}$-type supported on a $C^{\infty}$-smooth compact hypersurface $\Sigma \subset \mathbb{R}^{n}$, which splits $\mathbb{R}^{n}$ into a bounded open set $\Omega_{-}$and an unbounded open set $\Omega_{+}$. More precisely, denote by $\mathcal{A}_{ \pm}$the restrictions of $\mathcal{A}$ to $\Omega_{ \pm}$, let $\gamma^{ \pm}$and $\nu^{ \pm}$be the trace and conormal trace, respectively, on the boundary $\Sigma$ of $\Omega_{ \pm}$, let $\alpha, \beta \in C^{\infty}(\Sigma)$ be real functions with $\beta\left(x^{\prime}\right) \neq 0$ for all $x^{\prime} \in \Sigma$, and consider the elliptic realizations

$$
\begin{aligned}
& A_{\delta, \alpha} u=\mathcal{A}_{+} u_{+} \oplus \mathcal{A}_{-} u_{-}, \\
& \operatorname{dom} A_{\delta, \alpha}=\left\{u=u_{+} \oplus u_{-} \in H^{2}\left(\Omega_{+}\right) \oplus H^{2}\left(\Omega_{-}\right)\right. \text {: } \\
& \left.\gamma^{+} u_{+}=\gamma^{-} u_{-}, \nu^{+} u_{+}+\nu^{-} u_{-}=\alpha \gamma^{+} u_{+}\right\},
\end{aligned}
$$

and

$$
\begin{aligned}
A_{\delta^{\prime}, \beta} u= & \mathcal{A}_{+} u_{+} \oplus \mathcal{A}_{-} u_{-}, \\
\operatorname{dom} A_{\delta^{\prime}, \beta}=\left\{u=u_{+} \oplus u_{-} \in H^{2}\left(\Omega_{+}\right) \oplus H^{2}\left(\Omega_{-}\right):\right. & \\
& \left.\nu^{+} u_{+}+\nu^{-} u_{-}=0, \beta \nu^{+} u_{+}=\gamma^{+} u_{+}-\gamma^{-} u_{-}\right\},
\end{aligned}
$$

which are self-adjoint and bounded from below in $L_{2}\left(\mathbb{R}^{n}\right)$; cf. Theorem 2.1. Our main goal is to compare the resolvents of $A_{\delta, \alpha}$ and $A_{\delta^{\prime}, \beta}$ with the resolvent of the 'free' or 'unperturbed' self-adjoint realization

$$
A_{0} u=\mathcal{A} u, \quad \operatorname{dom} A_{0}=H^{2}\left(\mathbb{R}^{n}\right),
$$


and to prove spectral asymptotics formulae with refined remainder estimates for the singular values of the corresponding resolvent differences. Without loss of generality we may assume that a sufficiently large positive constant is added to $\mathcal{A}$ such that all operators under consideration have a positive lower bound; hence we consider

$$
G_{\delta, \alpha}=A_{\delta, \alpha}^{-1}-A_{0}^{-1} \quad \text { and } \quad G_{\delta^{\prime}, \beta}=A_{\delta^{\prime}, \beta}^{-1}-A_{0}^{-1} .
$$

It is known that both operators $G_{\delta, \alpha}$ and $G_{\delta^{\prime}, \beta}$ are compact in $L_{2}\left(\mathbb{R}^{n}\right)$, and estimates for the decay of the singular values $s_{k}\left(G_{\delta, \alpha}\right)$ and $s_{k}\left(G_{\delta^{\prime}, \beta}\right)$ were recently obtained in Behrndt et al. [6] and [7] (for the special case $\mathcal{A}=-\Delta+a$ ). In our main results Theorem 5.1 and Theorem 5.2 we shall prove the more precise asymptotic results with estimates of the remainder of the form

$$
\begin{aligned}
s_{k}\left(G_{\delta, \alpha}\right) & =C_{\delta, \alpha} k^{-\frac{3}{n-1}}+\mathrm{O}\left(k^{-\frac{4}{n-1}}\right), & & k \rightarrow \infty, \\
s_{k}\left(G_{\delta^{\prime}, \beta}\right) & =C_{\delta^{\prime}} k^{-\frac{2}{n-1}}+\mathrm{O}\left(k^{-\frac{3}{n-1}}\right), & & k \rightarrow \infty,
\end{aligned}
$$

with positive constants $C_{\delta, \alpha}$ and $C_{\delta^{\prime}}$ which are given explicitly in terms of the coefficients of $\mathcal{A}$ and $\alpha$; the constant $C_{\delta^{\prime}}$ is independent of $\beta$. Note that the singular values of $G_{\delta, \alpha}$ converge faster than the singular values of $G_{\delta^{\prime}, \beta}$. We mention that for the first result in (1.2) it is assumed that the function $\alpha$ does not vanish on $\Sigma$; if this assumption is dropped, the estimate holds with remainder o $\left(k^{-\frac{3}{n-1}}\right)$; cf. Theorem 5.1. In the course of our work we also make use of the direct sum $A_{\nu}$ of the self-adjoint Neumann operators in $L_{2}\left(\Omega_{+}\right)$ and $L_{2}\left(\Omega_{-}\right)$and we show that the singular values of $G_{\delta^{\prime}, \beta, \nu}=A_{\delta^{\prime}, \beta}^{-1}-A_{\nu}^{-1}$ satisfy

$$
s_{k}\left(G_{\delta^{\prime}, \beta, \nu}\right)=C_{\delta^{\prime}, \beta, \nu} k^{-\frac{3}{n-1}}+\mathrm{O}\left(k^{-\frac{4}{n-1}}\right), \quad k \rightarrow \infty,
$$

with the constant $C_{\delta^{\prime}, \beta, \nu}>0$ explicitly given; cf. Theorem 5.3. The proofs of (1.2) and (1.3) are mainly based on pseudodifferential techniques and classical results on spectral asymptotics of $\psi$ do's on closed $C^{\infty}$-smooth manifolds due to Seeley [47], Hörmander [37] and Grubb [28]. We also refer to Boutet de Monvel [11], Hörmander [38], Taylor [50], Rempel and Schulze [46] and Grubb [30, 31] for general pseudodifferential methods. A further ingredient in our analysis is a Krein-type resolvent formula, which provides a factorization of the operators in (1.1) and is discussed in detail in Section 4; cf. Brasche et al. [13], Alpay and Behrndt [1], Behrndt et al. [6, 7].

Our results in this paper contribute to a prominent field in the analysis of partial differential operators: asymptotic estimates for the resolvent difference of elliptic operators subject to different boundary conditions were first obtained by Povzner [45] and Birman [8]. These estimates were sharpened to spectral asymptotics formulae by Grubb [26] for bounded domains and by Birman and Solomjak [9, 10] for exterior domains, further generalized by Grubb [28, 29], and more recently in [32, 34, 36]; see also the review paper [35]. For the case of two Robin Laplacians a faster convergence of the singular values was observed in Behrndt et al. [5], and further refined to spectral asymptotics in Grubb [33]. We also list the closely related works Deift and Simon [16], Bardos et al. [3], Gorbachuk and Kutovoľ [25], Brasche [12], Carron [15], Malamud [43] and Lotoreichik and Rohleder [42] with spectral estimates for resolvent differences and resolvent power differences. 
We wish to emphasize that the operators $A_{\delta, \alpha}$ and $A_{\delta^{\prime}, \beta}$ have attracted considerable interest in the last two decades from more applied branches of mathematics and mathematical physics. In the special case $\mathcal{A}=-\Delta+a$ we refer to the review paper [18] by Exner for an overview on Schrödinger operators with $\delta$-interactions supported on curves and hypersurfaces. Such Hamiltonians are physically relevant in quantum mechanics, where they are employed in many-body problems and in the description of various nanostructures, as well as in the theory of photonic crystals; see, e.g. Figotin and Kuchment [24], Popov [44] and Brummelhuis and Duclos [14]. At the same time there is a mathematical motivation to study Schrödinger operators with $\delta$-interactions on hypersurfaces because these operators exhibit non-trivial and interesting spectral properties; for more details we refer to Brasche et al. [13], Exner et al. [19, 20, 22, 23], Suslina and Shterenberg [49], Kondej and Veselić [40], Kondej and Krejčiř́k [39], Duchêne and Raymond [17] and the references therein. Schrödinger operators with $\delta^{\prime}$-interactions supported on hypersurfaces are much less studied than their $\delta$-counterparts. They have been rigorously defined (in a general setting) only recently in [7]; the works Behrndt et al. [4] and Exner and Jex [21] on their spectral properties appeared subsequently. We also mention that for very special geometries such operators were considered earlier in Antoine et al. [2] and Shabani [48].

Acknowledgements. J. Behrndt and V. Lotoreichik gratefully acknowledge financial support by the Austrian Science Fund (FWF), project $\mathrm{P}$ 25162-N26. G. Grubb and M. Langer are grateful for the stimulating research stay and the hospitality at the Graz University of Technology in October 2013 where parts of this paper were written.

\section{The DIFFERENTIAL OPERATORS}

Throughout this paper let $\mathcal{A}$ be the following second-order formally symmetric differential expression on $\mathbb{R}^{n}$ :

$$
(\mathcal{A} u)(x):=-\sum_{j, k=1}^{n} \partial_{j}\left(a_{j k}(x) \partial_{k} u\right)(x)+a(x) u(x), \quad x \in \mathbb{R}^{n},
$$

with real-valued $a_{j k} \in C^{\infty}\left(\mathbb{R}^{n}\right)$ satisfying $a_{j k}(x)=a_{k j}(x)$ for all $x \in \mathbb{R}^{n}$, $j, k=1, \ldots, n$, and a bounded real-valued coefficient $a \in C^{\infty}\left(\mathbb{R}^{n}\right)$. We assume that $a_{j k}$ and all their derivatives are bounded and that $\mathcal{A}$ is uniformly strongly elliptic, i.e.

$$
\sum_{j, k=1}^{n} a_{j k}(x) \xi_{j} \xi_{k} \geq C|\xi|^{2}, \quad x, \xi \in \mathbb{R}^{n}
$$

for some constant $C>0$.

Further, let $\Sigma \subset \mathbb{R}^{n}$ be a $C^{\infty}$-smooth $(n-1)$-dimensional manifold that separates the Euclidean space $\mathbb{R}^{n}$ into a bounded open set $\Omega_{-}$and an unbounded open set $\Omega_{+}$. In the following we denote by $u_{+}$and $u_{-}$the restrictions of $u \in L_{2}\left(\mathbb{R}^{n}\right)$ to $\Omega_{+}$and $\Omega_{-}$, respectively; the restrictions of the differential expression $\mathcal{A}$ to $\Omega_{ \pm}$are denoted by $\mathcal{A}_{ \pm}$. For functions $u_{ \pm} \in H^{2}\left(\Omega_{ \pm}\right)$ 
denote by $\gamma^{ \pm} u_{ \pm}$the traces (boundary values on $\Sigma$ ) and by $\nu^{ \pm} u_{ \pm}$the outward conormal derivatives of $u_{ \pm}$:

$$
\nu^{ \pm} u_{ \pm}=\sum_{j, k=1}^{n} a_{j k} \nu_{ \pm, j} \gamma^{ \pm} \partial_{k} u_{ \pm},
$$

where $\left(\nu_{ \pm, 1}(x), \ldots, \nu_{ \pm, n}(x)\right)$ is the exterior unit normal to $\Omega_{ \pm}$at $x \in \Sigma$. If $u=u_{+} \oplus u_{-} \in H^{2}\left(\Omega_{+}\right) \oplus H^{2}\left(\Omega_{-}\right)$and $\gamma^{+} u_{+}=\gamma^{-} u_{-}$, then $u \in H^{1}\left(\mathbb{R}^{n}\right)$ and we write $\gamma u$ for $\gamma^{+} u_{+}=\gamma^{-} u_{-}$.

Let us introduce the following operators: the free realization of $\mathcal{A}$ in $L_{2}\left(\mathbb{R}^{n}\right)$,

$$
A_{0} u:=\mathcal{A} u, \quad \operatorname{dom} A_{0}:=H^{2}\left(\mathbb{R}^{n}\right),
$$

the Dirichlet realizations on $\Omega_{+}$and $\Omega_{-}$,

$$
A_{ \pm, \gamma} u_{ \pm}:=\mathcal{A}_{ \pm} u_{ \pm}, \quad \operatorname{dom} A_{ \pm, \gamma}:=\left\{u_{ \pm} \in H^{2}\left(\Omega_{ \pm}\right): \gamma^{ \pm} u_{ \pm}=0\right\},
$$

and the Neumann realizations,

$$
A_{ \pm, \nu} u_{ \pm}:=\mathcal{A}_{ \pm} u_{ \pm}, \quad \operatorname{dom} A_{ \pm, \nu}:=\left\{u_{ \pm} \in H^{2}\left(\Omega_{ \pm}\right): \nu^{ \pm} u_{ \pm}=0\right\} .
$$

It is well known that the operators $A_{0}, A_{+, \gamma}, A_{-, \gamma}, A_{+, \nu}$ and $A_{-, \nu}$ are selfadjoint and bounded below.

Let us also introduce direct sums of operators on $\Omega_{+}$and $\Omega_{-}$:

$$
A_{\gamma}:=A_{+, \gamma} \oplus A_{-, \gamma}, \quad A_{\nu}:=A_{+, \nu} \oplus A_{-, \nu},
$$

which are self-adjoint operators in $L_{2}\left(\mathbb{R}^{n}\right)=L_{2}\left(\Omega_{+}\right) \oplus L_{2}\left(\Omega_{-}\right)$. Note also that the domain of $A_{0}$ can be written with interface conditions:

$$
\begin{array}{r}
\operatorname{dom} A_{0}=\left\{u=u_{+} \oplus u_{-} \in H^{2}\left(\Omega_{+}\right) \oplus H^{2}\left(\Omega_{-}\right):\right. \\
\left.\gamma^{+} u_{+}=\gamma^{-} u_{-}, \quad \nu^{+} u_{+}=-\nu^{-} u_{-}\right\} .
\end{array}
$$

Moreover, let us fix a real-valued function $\alpha \in C^{\infty}(\Sigma)$, and define the $\delta$-operator with strength $\alpha$ by

$$
\begin{aligned}
A_{\delta, \alpha} u:= & \mathcal{A}_{+} u_{+} \oplus \mathcal{A}_{-} u_{-}, \\
\operatorname{dom} A_{\delta, \alpha}:= & \left\{u=u_{+} \oplus u_{-} \in H^{2}\left(\Omega_{+}\right) \oplus H^{2}\left(\Omega_{-}\right):\right. \\
& \left.\gamma^{+} u_{+}=\gamma^{-} u_{-}, \nu^{+} u_{+}+\nu^{-} u_{-}=\alpha \gamma u\right\} .
\end{aligned}
$$

Let us also fix a real-valued function $\beta \in C^{\infty}(\Sigma)$ such that $\beta$ is non-zero on $\Sigma$, and define the $\delta^{\prime}$-operator with strength $\beta$ by

$$
\begin{aligned}
A_{\delta^{\prime}, \beta} u:= & \mathcal{A}_{+} u_{+} \oplus \mathcal{A}_{-} u_{-}, \\
\operatorname{dom} A_{\delta^{\prime}, \beta}:= & \left\{u=u_{+} \oplus u_{-} \in H^{2}\left(\Omega_{+}\right) \oplus H^{2}\left(\Omega_{-}\right):\right. \\
& \left.\nu^{+} u_{+}+\nu^{-} u_{-}=0, \beta \nu^{+} u_{+}=\gamma^{+} u_{+}-\gamma^{-} u_{-}\right\} .
\end{aligned}
$$

The statements in the next theorem were shown in [6, Theorem 4.17] and [7, Theorem 3.11, 3.14, and 3.16] for the special case $\mathcal{A}=-\Delta+a$; the general case can be shown in a similar way. For the self-adjointness of $A_{\delta, \alpha}$ and $A_{\delta^{\prime}, \beta}$ one can also use the symmetry together with elliptic regularity theory as done in a related situation in [26, Theorem 7.3].

Theorem 2.1. The operators $A_{\delta, \alpha}$ and $A_{\delta^{\prime}, \beta}$ are self-adjoint and bounded below in $L_{2}\left(\mathbb{R}^{n}\right)$. 
Since all these operators are bounded below, we can assume without loss of generality (by adding a sufficiently large real constant to $a$ ) that $A_{0}, A_{ \pm, \gamma}$, $A_{ \pm, \nu}, A_{\delta, \alpha}$ and $A_{\delta^{\prime}, \beta}$ are positive with 0 in the resolvent set.

We shall often tacitly identify $H^{s}\left(\Omega_{+}\right) \oplus H^{s}\left(\Omega_{-}\right)$with $H^{s}\left(\Omega_{+}\right) \times H^{s}\left(\Omega_{-}\right)$ and write the operators in matrix form.

\section{Pseudodifferential methods}

In order to show the spectral asymptotics formulae we are aiming for, we have to go deeper into the definitions of the entering operators by pseudodifferential techniques. Pseudodifferential operators ( $\psi$ do's) $P$ are defined on $\mathbb{R}^{n}$ by formulae

$$
(P u)(x)=\operatorname{Op}(p(x, \xi)) u=p(x, D) u=\frac{1}{(2 \pi)^{n}} \int_{\mathbb{R}^{n}} e^{i x \cdot \xi} p(x, \xi) \hat{u}(\xi) \mathrm{d} x,
$$

where $\hat{u}(\xi)=\mathcal{F} u=\int e^{-i x \cdot \xi} u(x) \mathrm{d} x$ is the Fourier transform; $p(x, \xi)$ is called the symbol of $P$. There are various conditions on $p$, interpretations to distributions $u$, and rules of calculus, in particular behaviour under coordinate changes that allow the definition on manifolds, for which we refer to the vast literature, e.g. [38, 50, 31]. The symbols we consider are "classical" or "polyhomogeneous", meaning that $p(x, \xi)$ is an asymptotic series of functions $p_{d-j}(x, \xi), j=0,1,2, \ldots$, homogeneous of degree $d-j$ in $\xi ; p$ (and $P$ ) is then said to be of order $d$, and the principal symbol is the first term $p^{0}=p_{d}$. It has an invariant meaning in the manifold situation.

The following theorem is an important ingredient in the proofs in Section 5. The first part is essentially due to Seeley [47]. This paper treats the elliptic case; how the estimate can be extended to the general case is discussed, e.g. in [28, Lemma 4.5 and following paragraph] with more references given as well. The second part is due to Hörmander [37]. The transition between his formulation in terms of the counting function for $P^{-1}$ and the eigenvalue asymptotics for $P$ is accounted for, e.g. in [27, Lemma 6.2] and [30, Lemma A.5].

Theorem 3.1. Let $P$ be a classical pseudodifferential operator of negative order $-t$ on $\Sigma$, with principal symbol $p^{0}(x, \xi)$. Then the following statements hold.

(i) $P$ is a compact operator in $L_{2}(\Sigma)$, and its singular values satisfy

$$
s_{k}(P)=(c(P))^{\frac{t}{n-1}} k^{-\frac{t}{n-1}}+\mathrm{o}\left(k^{-\frac{t}{n-1}}\right), \quad k \rightarrow \infty,
$$

where

$$
c(P)=\frac{1}{(n-1)(2 \pi)^{n-1}} \int_{\Sigma} \int_{|\xi|=1}\left|p^{0}(x, \xi)\right|^{\frac{n-1}{t}} \mathrm{~d} \omega(\xi) \mathrm{d} \sigma(x) ;
$$

here $\sigma$ and $\omega$ are the surface measures on the hypersurfaces $\Sigma$ and $\left\{\xi \in \mathbb{R}^{n-1}:|\xi|=1\right\}$, respectively.

(ii) If, moreover, $P$ is elliptic and invertible, then the asymptotic estimate can be sharpened to the form

$$
s_{k}(P)=(c(P))^{\frac{t}{n-1}} k^{-\frac{t}{n-1}}+\mathrm{O}\left(k^{-\frac{t+1}{n-1}}\right), \quad k \rightarrow \infty .
$$


A general systematic theory covering the boundary value problems we are considering, as well as much more general situations, was introduced by Boutet de Monvel [11]: the theory of pseudodifferential boundary operators ( $\psi$ dbo's). Besides working with pseudodifferential operators $P$ on $\mathbb{R}^{n}$ and their versions $P_{+}$truncated to smooth subsets $\Omega$ (in particular to $\mathbb{R}_{+}^{n}$ ), the theory includes Poisson operators $K$ (going from $\partial \Omega$ to $\Omega$ ), trace operators $T$ (going from $\Omega$ to $\partial \Omega$ ), $\psi$ do's $S$ on $\partial \Omega$, and the so-called singular Green operators $G$, essentially in the form of finite sums or infinite series: $\sum K_{j} T_{j}$. We shall not use the $\psi$ dbo calculus in full generality, but rather its notation and elementary composition rules. Details on the $\psi$ dbo calculus are found, e.g. in $[11,46,28,30,31]$.

In this theory, the operators are described by use of local coordinate systems, carrying the study of the operators over to the situation of $\Omega=$ $\mathbb{R}_{+}^{n}:=\mathbb{R}^{n-1} \times \mathbb{R}_{+}$. Here a differential operator $P=\sum_{|\alpha| \leq m} a_{\alpha}(x) D^{\alpha}$ with the symbol $p(x, \xi)=\sum_{|\alpha| \leq m} a_{\alpha}(x) \xi^{\alpha}$ has the principal symbol $p^{0}(x, \xi)=$ $\sum_{|\alpha|=m} a_{\alpha}(x) \xi^{\alpha}$, and the model operator at a point $\left(x^{\prime}, 0\right) \in \mathbb{R}^{n-1} \times\{0\}$ is $p^{0}\left(x^{\prime}, 0, \xi^{\prime}, D_{n}\right)=\sum_{|\alpha|=m} a_{\alpha}\left(x^{\prime}, 0\right) \xi^{\prime \alpha^{\prime}} D_{n}^{\alpha_{n}}$. The solution operator for the Dirichlet problem for our $\mathcal{A}$ on $\Omega_{+}$with non-zero boundary data, zero interior data, is a Poisson operator. Such operators, carried over to $\mathbb{R}_{+}^{n}$, are generally of the form

$$
(K \varphi)(x)=\frac{1}{(2 \pi)^{n-1}} \int_{\mathbb{R}^{n-1}} e^{i x^{\prime} \cdot \xi^{\prime}} \tilde{k}\left(x^{\prime}, x_{n}, \xi^{\prime}\right) \hat{\varphi}\left(\xi^{\prime}\right) \mathrm{d} \xi^{\prime}, \quad x=\left(x^{\prime}, x_{n}\right),
$$

where $\tilde{k}\left(x^{\prime}, x_{n}, \xi^{\prime}\right)$ is called the symbol-kernel of $K$; it is a $C^{\infty}$-function on $\mathbb{R}_{+}^{n} \times \mathbb{R}^{n-1}$ that is rapidly decreasing for $x_{n} \rightarrow \infty$, with

$$
\begin{aligned}
\sup _{x_{n} \in(0, \infty)}\left|x_{n}^{l} \partial_{n}^{l^{\prime}} \partial_{x^{\prime}}^{\beta} \partial_{\xi^{\prime}}^{\alpha} \tilde{k}\left(x, \xi^{\prime}\right)\right| \leq C_{l, l^{\prime}, \alpha, \beta}\left(1+\left|\xi^{\prime}\right|\right)^{d-l+l^{\prime}-|\alpha|}, & \\
& \quad l, l^{\prime} \in \mathbb{N}_{0}, \alpha, \beta \in \mathbb{N}_{0}^{n-1},
\end{aligned}
$$

for some $d$, and is then said to be of order $d$. More information on the structure of symbol-kernels is found, e.g. in [31, Section 10.1]. The symbol-kernel $\tilde{k}$ is a series of terms with certain quasi-homogeneities in $\left(\xi^{\prime}, x_{n}\right)$, corresponding to falling homogeneities in $\xi$ of the terms in the Fourier transform w.r.t. $x_{n}$, the symbol $k=\mathcal{F}_{x_{n} \rightarrow \xi_{n}} e^{+} \tilde{k}$. There is a principal part, the top order term.

It is also known from the general calculus that the adjoint of a Poisson operator is a trace operator (of the general form defined in the $\psi$ dbo calculus), and that a Poisson operator composed to the left with a trace operator gives a pseudodifferential operator on $\partial \Omega$ (on $\mathbb{R}^{n-1}$ in local coordinates).

In Lemma 3.2 below we describe the principal symbol-kernel of the Poisson solution operators and the principal symbols of the Dirichlet-to-Neumann and Neumann-to-Dirichlet maps corresponding to $\mathcal{A}$ from (2.1) both on $\Omega_{+}$ and on $\Omega_{-}$. For this purpose, let us write the principal symbol $a^{0}(x, \xi)$ of $\mathcal{A}$ in local coordinates $\left(x^{\prime}, x_{n}\right)=\left(x_{1}, \ldots, x_{n-1}, x_{n}\right) \in \mathbb{R}_{+}^{n}$ at the boundary of $\Omega_{+}$:

$$
\underline{a}^{0}\left(x^{\prime}, 0, \xi\right)=\sum_{j, k=1}^{n} \underline{a}_{j k}\left(x^{\prime}\right) \xi_{j} \xi_{k}=\underline{a}_{n n}\left(x^{\prime}\right) \xi_{n}^{2}+2 b\left(x^{\prime}, \xi^{\prime}\right) \xi_{n}+c\left(x^{\prime}, \xi^{\prime}\right) .
$$


Here $\xi^{\prime}=\left(\xi_{1}, \ldots, \xi_{n-1}\right), \underline{a}_{n n}\left(x^{\prime}\right)>0$,

$$
b\left(x^{\prime}, \xi^{\prime}\right):=\sum_{j=1}^{n-1} \underline{a}_{j n}\left(x^{\prime}\right) \xi_{j}, \quad c\left(x^{\prime}, \xi^{\prime}\right):=\sum_{j, k=1}^{n-1} \underline{a}_{j k}\left(x^{\prime}\right) \xi_{j} \xi_{k}
$$

and $\underline{a}_{n n} c>b^{2}$ when $\xi^{\prime} \neq 0$ since $\underline{a}^{0}>0$ for $\xi \neq 0$. The roots of the second-order polynomial in $\xi_{n}$ on the right-hand side of (3.2) are

$$
\lambda_{ \pm}\left(x^{\prime}, \xi^{\prime}\right)=\frac{-b\left(x^{\prime}, \xi^{\prime}\right) \pm i \sqrt{\underline{a}_{n n}\left(x^{\prime}\right) c\left(x^{\prime}, \xi^{\prime}\right)-\left(b\left(x^{\prime}, \xi^{\prime}\right)\right)^{2}}}{\underline{a}_{n n}\left(x^{\prime}\right)}
$$

lying in the upper, respectively lower, complex half-plane and being homogeneous of degree 1 in $\xi^{\prime}$. Define

$$
\begin{aligned}
\kappa_{0} & :=\sqrt{\underline{a}_{n n} c-b^{2}} \quad\left(>0 \text { when } \xi^{\prime} \neq 0\right), \\
\kappa_{ \pm} & :=\mp i \lambda_{ \pm}=\frac{\kappa_{0} \pm i b}{\underline{a}_{n n}} .
\end{aligned}
$$

Clearly, $\kappa_{ \pm}$are complex conjugates and have positive real part: $\operatorname{Re} \kappa_{ \pm}=$ $\kappa_{0} / \underline{a}_{n n}$, and satisfy $\kappa_{+} \bar{\kappa}_{+}=c / \underline{a}_{n n}$. With these expressions we can factorize $\underline{a}^{0}$ :

$$
\begin{aligned}
\underline{a}^{0}\left(x^{\prime}, 0, \xi^{\prime}, \xi_{n}\right) & =\underline{a}_{n n}\left(x^{\prime}\right)\left(\kappa_{+}+i \xi_{n}\right)\left(\kappa_{-}-i \xi_{n}\right), \\
\underline{a}^{0}\left(x^{\prime}, 0, \xi^{\prime}, D_{n}\right) & =\underline{a}_{n n}\left(x^{\prime}\right)\left(\kappa_{+}+\partial_{n}\right)\left(\kappa_{-}-\partial_{n}\right) .
\end{aligned}
$$

Let $K_{\gamma}^{ \pm}: H^{3 / 2}(\Sigma) \rightarrow H^{2}\left(\Omega_{ \pm}\right)$be the Poisson solution operators that map a $\varphi \in H^{3 / 2}\left(\Omega_{ \pm}\right)$onto the solutions $u_{ \pm} \in H^{2}\left(\Omega_{ \pm}\right)$of the boundary value problems

$$
\mathcal{A}_{ \pm} u_{ \pm}=0, \quad \gamma^{ \pm} u_{ \pm}=\varphi
$$

Similarly, let $K_{\nu}^{ \pm}: H^{1 / 2}(\Sigma) \rightarrow H^{2}\left(\Omega_{ \pm}\right)$be the Poisson solution operators corresponding to the Neumann problems

$$
\mathcal{A}_{ \pm} u_{ \pm}=0, \quad \nu^{ \pm} u_{ \pm}=\psi .
$$

Moreover, we define the Dirichlet-to-Neumann and Neumann-to-Dirichlet operators by

$$
P_{\gamma, \nu}^{ \pm}:=\nu^{ \pm} K_{\gamma}^{ \pm}, \quad P_{\nu, \gamma}^{ \pm}:=\gamma^{ \pm} K_{\nu}^{ \pm}
$$

In the next lemma we collect properties of these operators, which are needed in the proofs of our main results.

Lemma 3.2. Let the operators $K_{\gamma}^{ \pm}, K_{\nu}^{ \pm}, P_{\gamma, \nu}^{ \pm}$and $P_{\nu, \gamma}^{ \pm}$be as above. Then the following statements hold.

(i) The operators $K_{\gamma}^{+}$and $K_{\nu}^{+}$are Poisson operators of orders 0 and -1 , respectively. Their principal symbol-kernels are, in local coordinates,

$$
\begin{aligned}
& \tilde{k}_{\gamma}^{+0}\left(x^{\prime}, x_{n}, \xi^{\prime}\right)=e^{-\kappa_{+}\left(x^{\prime}, \xi^{\prime}\right) x_{n}}, \\
& \tilde{k}_{\nu}^{+0}\left(x^{\prime}, x_{n}, \xi^{\prime}\right)=\frac{1}{\kappa_{0}\left(x^{\prime}, \xi^{\prime}\right)} e^{-\kappa_{+}\left(x^{\prime}, \xi^{\prime}\right) x_{n}} .
\end{aligned}
$$

(ii) The operators $P_{\gamma, \nu}^{+}$and $P_{\nu, \gamma}^{+}$are pseudodifferential operators of orders 1 and -1 , respectively. Their principal symbols are

$$
p_{\gamma, \nu}^{+0}\left(x^{\prime}, \xi^{\prime}\right)=\kappa_{0}\left(x^{\prime}, \xi^{\prime}\right) \quad \text { and } \quad p_{\nu, \gamma}^{+0}\left(x^{\prime}, \xi^{\prime}\right)=\frac{1}{\kappa_{0}\left(x^{\prime}, \xi^{\prime}\right)},
$$


which are positive.

(iii) The compositions $\left(K_{\gamma}^{+}\right)^{*} K_{\gamma}^{+}$and $\left(K_{\nu}^{+}\right)^{*} K_{\nu}^{+}$are pseudodifferential operators of orders -1 and -3 , respectively. Their principal symbols are

$$
\frac{\underline{a}_{n n}\left(x^{\prime}\right)}{2 \kappa_{0}\left(x^{\prime}, \xi^{\prime}\right)} \quad \text { and } \quad \frac{\underline{a}_{n n}\left(x^{\prime}\right)}{2\left(\kappa_{0}\left(x^{\prime}, \xi^{\prime}\right)\right)^{3}} .
$$

For $\mathcal{A}$ on $\Omega_{-}$the formulae hold with $\kappa_{-}$instead of $\kappa_{+}$.

Proof. (i) To find the principal symbol-kernel of $K_{\gamma}^{+}$, we have to solve the following model problem for each $\left(x^{\prime}, \xi^{\prime}\right)$ with $\xi^{\prime} \neq 0$ on the one-dimensional level:

$$
\underline{a}^{0}\left(x^{\prime}, 0, \xi^{\prime}, D_{n}\right) u\left(x_{n}\right)=0 \quad \text { on } \mathbb{R}_{+}, \quad u(0)=\varphi \in \mathbb{C},
$$

where $\underline{a}^{0}$ is the principal symbol of $\mathcal{A}$ in local coordinates. It follows from (3.6) and the inequality $\operatorname{Re} \kappa_{+}>0$ that the $L_{2}\left(\mathbb{R}_{+}\right)$-solution of (3.13) is

$$
u\left(x_{n}\right)=\varphi e^{-\kappa+x_{n}} .
$$

Hence $\tilde{k}_{\gamma}^{+0}=e^{-\kappa_{+} x_{n}}$ is the principal symbol-kernel of the Poisson operator $K_{\gamma}^{+}$. This shows (3.10). In view of (3.1), it has order 0 .

Let us now consider the Neumann problem. In local coordinates the conormal derivative takes the form

$$
\underline{\nu}^{+} u=-\left.\underline{a}_{n n} \partial_{x_{n}} u\right|_{x_{n}=0}-\sum_{k=1}^{n-1} \underline{a}_{n k} i \xi_{k} u(0)
$$

cf. (2.2) and observe that the outward normal is $(0, \ldots, 0,-1)$. Since

$$
\underline{\nu}^{+} e^{-\kappa+x_{n}}=-\underline{a}_{n n}\left(-\kappa_{+}\right)-\sum_{k=1}^{n-1} \underline{a}_{n k} i \xi_{k}=\underline{a}_{n n} \frac{\kappa_{0}+i b}{\underline{a}_{n n}}-i b=\kappa_{0},
$$

the solution of $\underline{a}^{0}\left(x^{\prime}, 0, \xi^{\prime}, D_{n}\right) u\left(x_{n}\right)=0, \underline{\nu}^{+} u=\psi$ is

$$
u\left(x_{n}\right)=\psi \frac{1}{\kappa_{0}} e^{-\kappa_{+} x_{n}},
$$

which yields (3.11). Moreover, $\tilde{k}_{\nu}^{+0}$ has order -1 .

(ii) It follows from (3.14) that the Dirichlet-to-Neumann operator $P_{\gamma, \nu}^{+}$ has the principal symbol $p_{\gamma, \nu}^{+0}=\underline{\nu}^{+} \tilde{k}_{\gamma}^{+0}=\kappa_{0}$, which is of order 1 since $\kappa_{0}$ is homogeneous of degree 1 in $\xi^{\prime}$. The principal symbol of $P_{\nu, \gamma}^{+}$is $p_{\nu, \gamma}^{+0}=$ $\left.\tilde{k}_{\nu}^{+0}\right|_{x_{n}=0}=1 / \kappa_{0}$. like

(iii) The adjoint of $K_{\gamma}^{+}$has the principal part in local coordinates acting

$$
u(x) \mapsto \frac{1}{(2 \pi)^{n-1}} \int_{\mathbb{R}^{n-1}} e^{i x^{\prime} \cdot \xi^{\prime}} \int_{0}^{\infty} e^{-\bar{\kappa}_{+} x_{n}} \mathcal{F}_{x^{\prime} \rightarrow \xi^{\prime}} u\left(x^{\prime}, x_{n}\right) \mathrm{d} x_{n} \mathrm{~d} \xi^{\prime},
$$

with symbol-kernel $\overline{\tilde{k}_{\gamma}^{+0}}$ and order -1 ; for the latter see, e.g. [31, Theorem 10.29 and Remark 10.6]. Then the composition of the principal part of $\left(K_{\gamma}^{+}\right)^{*}$ with the principal part of $K_{\gamma}^{+}$is the $\psi$ do on $\mathbb{R}^{n-1}$ with symbol

$$
\int_{0}^{\infty} e^{-\bar{\kappa}_{+} x_{n}} e^{-\kappa_{+} x_{n}} \mathrm{~d} x_{n}=\frac{1}{\bar{\kappa}_{+}+\kappa_{+}}=\frac{\underline{a}_{n n}}{2 \kappa_{0}}
$$

and order -1 by [31, Proposition $10.10(\mathrm{v})]$. 
For $K_{\nu}^{+}$, the principal part of the adjoint acts like

$$
u(x) \mapsto \frac{1}{(2 \pi)^{n-1}} \int_{\mathbb{R}^{n-1}} e^{i x^{\prime} \cdot \xi^{\prime}} \int_{0}^{\infty} \frac{1}{\kappa_{0}} e^{-\bar{\kappa}+x_{n}} \mathcal{F}_{x^{\prime} \rightarrow \xi^{\prime}} u\left(x^{\prime}, x_{n}\right) \mathrm{d} x_{n} \mathrm{~d} \xi^{\prime},
$$

so we find that $\left(K_{\nu}^{+}\right)^{*} K_{\nu}^{+}$has the principal symbol

$$
\int_{0}^{\infty} \frac{1}{\kappa_{0}} e^{-\bar{\kappa}_{+} x_{n}} \frac{1}{\kappa_{0}} e^{-\kappa_{+} x_{n}} \mathrm{~d} x_{n}=\frac{1}{\kappa_{0}^{2}} \cdot \frac{1}{\bar{\kappa}_{+}+\kappa_{+}}=\frac{\underline{a}_{n n}}{2 \kappa_{0}^{3}} .
$$

For invariance with respect to coordinate changes see, e.g. [31, Theorem 8.1] for $\psi$ do's and [30, Theorem 2.4.11] for $\psi$ dbo's.

For the same operator $\mathcal{A}$ considered on $\Omega_{-}$, the symbol in local coordinates at a boundary point is as above with the direction of $x_{n}$, and hence also of $\xi_{n}$, reverted. Then $\kappa_{+}$and $\kappa_{-}$exchange roles.

The following lemma collects some properties of the operators $K_{\gamma}^{ \pm}$and $K_{\nu}^{ \pm}$considered as operators between $L_{2}$-spaces.

Lemma 3.3. Let $K_{\gamma}^{ \pm}$and $K_{\nu}^{ \pm}$be as above. Then the $L_{2}$-adjoints of $K_{\gamma}^{ \pm}$ and $K_{\nu}^{ \pm}$satisfy

$$
\left(K_{\gamma}^{ \pm}\right)^{*}=-\nu^{ \pm} A_{ \pm, \gamma}^{-1}, \quad\left(K_{\nu}^{ \pm}\right)^{*}=\gamma^{ \pm} A_{ \pm, \nu}^{-1} .
$$

Proof. Let $\varphi \in H^{3 / 2}(\Sigma)$ and set $u_{+}:=K_{\gamma}^{+} \varphi$, which satisfies (3.7). Moreover, let $f \in L_{2}\left(\Omega_{+}\right)$and set $v_{+}:=A_{+, \gamma}^{-1} f$. Then Green's identity implies

$$
\begin{aligned}
\left(f, K_{\gamma}^{+} \varphi\right) & =\left(\mathcal{A}_{+} v_{+}, u_{+}\right)=\left(\mathcal{A}_{+} v_{+}, u_{+}\right)-\left(v_{+}, \mathcal{A}_{+} u_{+}\right) \\
& =\left(\gamma^{+} v_{+}, \nu^{+} u_{+}\right)-\left(\nu^{+} v_{+}, \gamma^{+} u_{+}\right)=-\left(\nu^{+} A_{+, \gamma}^{-1} f, \varphi\right)
\end{aligned}
$$

which yields the relation for $K_{\gamma}^{+}$in (3.15) since $\nu^{+} A_{+, \gamma}^{-1}$ is bounded. The other relations in (3.15) are shown in a similar way.

Example 3.4. In the special, important case $\mathcal{A}=-\Delta$ we derive from (3.2) and (3.3) that

$$
\underline{a}_{n n}\left(x^{\prime}\right) \equiv 1, \quad b\left(x^{\prime}, \xi^{\prime}\right) \equiv 0, \quad c\left(x^{\prime}, \xi^{\prime}\right)=\left|\xi^{\prime}\right|^{2} ;
$$

note that the principal symbol at the boundary point is unchanged under the transformation to local coordinates. Hence, following (3.4) we get

$$
\kappa_{ \pm}\left(x^{\prime}, \xi^{\prime}\right)=\kappa_{0}\left(x^{\prime}, \xi^{\prime}\right)=\left|\xi^{\prime}\right| .
$$

Thus, according to Lemma 3.2 the principal symbol-kernels of $K_{\gamma}^{ \pm}, K_{\nu}^{ \pm}$are

$$
\tilde{k}_{\gamma}^{ \pm 0}=e^{-\left|\xi^{\prime}\right| x_{n}} \quad \text { and } \quad \tilde{k}_{\nu}^{ \pm 0}=\frac{e^{-\left|\xi^{\prime}\right| x_{n}}}{\left|\xi^{\prime}\right|} ;
$$

the principal symbols of $P_{\gamma, \nu}^{ \pm}, P_{\nu, \gamma}^{ \pm}$are

$$
p_{\gamma, \nu}^{ \pm 0}=\left|\xi^{\prime}\right| \quad \text { and } \quad p_{\nu, \gamma}^{ \pm 0}=\frac{1}{\left|\xi^{\prime}\right|} ;
$$

and the principal symbols of $\left(K_{\gamma}^{ \pm}\right)^{*} K_{\gamma}^{ \pm},\left(K_{\nu}^{ \pm}\right)^{*} K_{\nu}^{ \pm}$are

$$
\frac{1}{2\left|\xi^{\prime}\right|} \quad \text { and } \quad \frac{1}{2\left|\xi^{\prime}\right|^{3}} \text {. }
$$




\section{KREIN-TYPE FORMULAE}

In this section we provide Krein-type formulae for differences between inverses of self-adjoint realizations of $\mathcal{A}$ defined in Section 2. First we derive Krein-type formulae in a general setting, and then we simplify these formulae for particular $\delta$ and $\delta^{\prime}$-couplings. Similar formulae for systems acting on a single bounded domain can be found in the paper [26]. For coupled problems the reader may also consult [6, Section 4] and [7, Section 3].

Let us define the trace mapping

$$
\varrho: H^{2}\left(\Omega_{+}\right) \oplus H^{2}\left(\Omega_{-}\right) \rightarrow H^{3 / 2}(\Sigma) \times H^{3 / 2}(\Sigma) \times H^{1 / 2}(\Sigma) \times H^{1 / 2}(\Sigma)
$$

by

$$
\varrho\left(u_{+} \oplus u_{-}\right):=\left(\begin{array}{l}
\gamma^{+} u_{+} \\
\gamma^{-} u_{-} \\
\nu^{+} u_{+} \\
\nu^{-} u_{-}
\end{array}\right),
$$

where $\gamma^{ \pm}$are the traces on $\Sigma$ from the two sides of it, and $\nu^{ \pm}$are the outward conormal derivatives; cf. Section 2. Note that the map $\varrho$ is surjective by the classical trace theorem; see, e.g. [41].

Let in the following $\alpha, \beta \in C^{\infty}(\Sigma)$ be real-valued with $\beta$ non-vanishing on $\Sigma$. We consider realizations of $\mathcal{A}$ in $L_{2}\left(\mathbb{R}^{n}\right)$ defined by

$$
\begin{aligned}
A_{*} u & :=\mathcal{A}_{+} u_{+} \oplus \mathcal{A}_{-} u_{-}, \\
\operatorname{dom} A_{*} & :=\left\{u \in H^{2}\left(\Omega_{+}\right) \oplus H^{2}\left(\Omega_{-}\right): B_{*} \varrho u=0\right\},
\end{aligned}
$$

where $B_{*}$ is one of the following matrices

$$
\begin{aligned}
& B_{0}=\left(\begin{array}{cccc}
1 & -1 & 0 & 0 \\
0 & 0 & 1 & 1
\end{array}\right), B_{\gamma}=\left(\begin{array}{cccc}
1 & 0 & 0 & 0 \\
0 & 1 & 0 & 0
\end{array}\right), B_{\nu}=\left(\begin{array}{llll}
0 & 0 & 1 & 0 \\
0 & 0 & 0 & 1
\end{array}\right), \\
& B_{\delta, \alpha}=\left(\begin{array}{cccc}
1 & -1 & 0 & 0 \\
-\alpha & 0 & 1 & 1
\end{array}\right), B_{\delta^{\prime}, \beta}=\left(\begin{array}{cccc}
1 & -1 & -\beta & 0 \\
0 & 0 & 1 & 1
\end{array}\right) .
\end{aligned}
$$

The self-adjoint operators $A_{0}, A_{\gamma}, A_{\nu}, A_{\delta, \alpha}$ and $A_{\delta^{\prime}, \beta}$ defined in (2.3), (2.4), (2.5) and (2.6) correspond, respectively, to $B_{0}, B_{\gamma}, B_{\nu}, B_{\delta, \alpha}$ and $B_{\delta^{\prime}, \beta}$, in the sense of (4.3). The boundary conditions that are induced by the matrices $B_{0}, B_{\gamma}, B_{\nu}, B_{\delta, \alpha}$ and $B_{\delta^{\prime}, \beta}$ are analogues of what are called normal boundary conditions in [26]. A typical property of such boundary conditions is given in the lemma below, which follows from the fact that $\varrho$ defined in (4.1), (4.2) is surjective.

Lemma 4.1. With @ defined by (4.1) and (4.2), let $B_{*}$ be one of the matrices in (4.4) and let us define

$$
R_{*}:=H^{s}(\Sigma) \times H^{t}(\Sigma)
$$

with

$$
\begin{array}{ll}
s=3 / 2, t=1 / 2 & \text { if } B_{*}=B_{0}, B_{\delta, \alpha}, \text { or } B_{\delta^{\prime}, \beta}, \\
s=t=3 / 2 & \text { if } B_{*}=B_{\gamma}, \\
s=t=1 / 2 & \text { if } B_{*}=B_{\nu} .
\end{array}
$$

Then $B_{*} \varrho$ is surjective from $H^{2}\left(\Omega_{+}\right) \oplus H^{2}\left(\Omega_{-}\right)$onto $R_{*}$. 
As discussed at the end of Section 2 we can assume without loss of generality that the operators $A_{0}, A_{\gamma}, A_{\nu}, A_{\delta, \alpha}$ and $A_{\delta^{\prime}, \beta}$ are positive with 0 in the resolvent set. Then the semi-homogeneous Dirichlet and Neumann boundary value problems (3.7) and (3.8) for the differential expression $\mathcal{A}_{ \pm}$on $\Omega_{ \pm}$ are uniquely solvable in $H^{2}\left(\Omega_{ \pm}\right)$, and $K_{\gamma}^{ \pm}$and $K_{\nu}^{ \pm}$are the corresponding solution operators. It follows that for all $\varphi=\left(\begin{array}{l}\varphi_{+} \\ \varphi_{-}\end{array}\right) \in H^{3 / 2}(\Sigma) \times H^{3 / 2}(\Sigma)$ the problem

$$
\mathcal{A}_{+} u_{+} \oplus \mathcal{A}_{-} u_{-}=0, \quad B_{\gamma} \varrho u=\varphi,
$$

with $B_{\gamma}$ as in (4.4), has a unique solution $u=u_{+} \oplus u_{-} \in H^{2}\left(\Omega_{+}\right) \oplus H^{2}\left(\Omega_{-}\right)$. The corresponding solution operator is

$$
K_{\gamma}=\left(\begin{array}{cc}
K_{\gamma}^{+} & 0 \\
0 & K_{\gamma}^{-}
\end{array}\right)
$$

i.e. $u=K_{\gamma} \varphi$. Note that for $u=u_{+} \oplus u_{-} \in H^{2}\left(\Omega_{+}\right) \oplus H^{2}\left(\Omega_{-}\right)$one has

$$
\mathcal{A}_{+} u_{+} \oplus \mathcal{A}_{-} u_{-}=0 \quad \Rightarrow \quad u=K_{\gamma} B_{\gamma} \varrho u .
$$

Similarly, for all $\psi=\left(\begin{array}{c}\psi_{+} \\ \psi_{-}\end{array}\right) \in H^{1 / 2}(\Sigma) \times H^{1 / 2}(\Sigma)$ the problem

$$
\mathcal{A}_{+} u_{+} \oplus \mathcal{A}_{-} u_{-}=0, \quad B_{\nu} \varrho u=\psi,
$$

with $B_{\nu}$ as in (4.4), has a unique solution $u=u_{+} \oplus u_{-} \in H^{2}\left(\Omega_{+}\right) \oplus H^{2}\left(\Omega_{-}\right)$; the solution operator is given by

$$
K_{\nu}=\left(\begin{array}{cc}
K_{\nu}^{+} & 0 \\
0 & K_{\nu}^{-}
\end{array}\right) .
$$

In the next proposition we investigate the solvability of the boundary value problems associated with the matrices $B_{*}$ in (4.4) and derive related properties of the $2 \times 2$ matrix $\psi$ do's

$$
\Phi_{*}:=B_{*} \varrho K_{\gamma} \quad \text { and } \quad \Psi_{*}:=B_{*} \varrho K_{\nu} .
$$

Proposition 4.2. Let $B_{*}$ be one of the matrices in (4.4) with the associated space $R_{*}$ as in (4.5), and let the matrix $\psi d o$ 's $\Phi_{*}$ and $\Psi_{*}$ be defined by (4.9). Then the following statements hold.

(i) For all $\psi \in R_{*}$ the boundary value problem

$$
\mathcal{A}_{+} u_{+} \oplus \mathcal{A}_{-} u_{-}=0, \quad B_{*} \varrho u=\psi,
$$

has a unique solution $u=u_{+} \oplus u_{-} \in H^{2}\left(\Omega_{+}\right) \oplus H^{2}\left(\Omega_{-}\right)$.

(ii) The matrix $\psi$ do $\Phi_{*}$ is bijective from $H^{3 / 2}(\Sigma) \times H^{3 / 2}(\Sigma)$ onto $R_{*}$.

(iii) The matrix $\psi$ do $\Psi_{*}$ is bijective from $H^{1 / 2}(\Sigma) \times H^{1 / 2}(\Sigma)$ onto $R_{*}$.

Proof. In the following, $A_{*}$ is the self-adjoint operator in $L_{2}\left(\mathbb{R}^{n}\right)$ corresponding to the matrix $B_{*}$ in the sense of (4.3). By our assumptions $A_{*}$ is strictly positive. This implies that the semi-homogeneous boundary value problem

$$
\mathcal{A}_{+} w_{+} \oplus \mathcal{A}_{-} w_{-}=f, \quad B_{*} \varrho w=0,
$$

is uniquely solvable for all $f \in L_{2}\left(\mathbb{R}^{n}\right)$, and the unique solution is given by $w:=A_{*}^{-1} f \in H^{2}\left(\Omega_{+}\right) \oplus H^{2}\left(\Omega_{-}\right)$.

(i) Let $\psi \in R_{*}$ and choose $v \in H^{2}\left(\Omega_{+}\right) \oplus H^{2}\left(\Omega_{-}\right)$such that $B_{*} \varrho v=\psi$, which is possible by Lemma 4.1. The boundary value problem

$$
\mathcal{A}_{+} z_{+} \oplus \mathcal{A}_{-} z_{-}=-\mathcal{A}_{+} v_{+} \oplus \mathcal{A}_{-} v_{-}, \quad B_{*} \varrho z=0,
$$


has a unique solution $z \in H^{2}\left(\Omega_{+}\right) \oplus H^{2}\left(\Omega_{-}\right)$; cf. (4.11). It follows that

$$
u:=z+v \in H^{2}\left(\Omega_{+}\right) \oplus H^{2}\left(\Omega_{-}\right)
$$

is a solution of (4.10). Moreover, this solution is unique. In fact, suppose that $\tilde{u} \in H^{2}\left(\Omega_{+}\right) \oplus H^{2}\left(\Omega_{-}\right)$is also a solution of (4.10). Then $u-\widetilde{u} \in \operatorname{dom} A_{*}$ and $A_{*}(u-\widetilde{u})=0$. As $A_{*}$ is strictly positive we conclude that $u=\widetilde{u}$.

(ii) First we verify that $\Phi_{*}$ is injective. Suppose that $\Phi_{*} \varphi=B_{*} \varrho K_{\gamma} \varphi=0$ holds for some $\varphi \in H^{3 / 2}(\Sigma) \times H^{3 / 2}(\Sigma), \varphi \neq 0$. Then the function $K_{\gamma} \varphi \neq 0$ belongs to $\operatorname{dom} A_{*}$ and satisfies $A_{*} K_{\gamma} \varphi=0$, a contradiction to the strict positivity of $A_{*}$. In order to show that $\Phi_{*}$ is surjective, let $\psi \in R_{*}$. By item (i) the boundary value problem

$$
\mathcal{A}_{+} u_{+} \oplus \mathcal{A}_{-} u_{-}=0, \quad B_{*} \varrho u=\psi,
$$

has a unique solution $u \in H^{2}\left(\Omega_{+}\right) \oplus H^{2}\left(\Omega_{-}\right)$. Define

$$
\varphi:=B_{\gamma} \varrho u \in H^{3 / 2}(\Sigma) \times H^{3 / 2}(\Sigma) .
$$

Note that by item (i) the boundary value problem

$$
\mathcal{A}_{+} v_{+} \oplus \mathcal{A}_{-} v_{-}=0, \quad B_{\gamma} \varrho v=\varphi
$$

has a unique solution in $H^{2}\left(\Omega_{+}\right) \oplus H^{2}\left(\Omega_{-}\right)$. Observe that $K_{\gamma} \varphi$ and $u$ both are solutions of the above problem. Hence $u=K_{\gamma} \varphi$ by the uniqueness. From

$$
\Phi_{*} \varphi=B_{*} \varrho K_{\gamma} \varphi=B_{*} \varrho u=\psi
$$

we conclude that $\psi \in \operatorname{ran} \Phi_{*}$. It follows that $\Phi_{*}$ is surjective onto $R_{*}$.

(iii) The proof of this item is analogous to the proof of (ii).

We mention that the matrix $\psi$ do's $\Phi_{*}$ and $\Psi_{*}$ in (4.9) are elliptic. This is essentially a consequence of the bijectivity shown in Proposition 4.2 above.

In the next theorem we give Krein-type factorizations for differences of inverses between either $A_{\gamma}$ or $A_{\nu}$ and one of the operators $A_{0}, A_{\nu}, A_{\delta, \alpha}$ and $A_{\delta^{\prime}, \beta}$.

Theorem 4.3. Let $B_{*}$ be one of the matrices in (4.4), let $A_{*}, A_{\gamma}$ and $A_{\nu}$ be the self-adjoint operators in $L_{2}\left(\mathbb{R}^{n}\right)$ corresponding to the matrices $B_{*}, B_{\gamma}$ and $B_{\nu}$ as in (4.3), and let $\varrho$ be the trace map in (4.1), (4.2). Then the following statements are true.

(i) The formula

$$
A_{*}^{-1}-A_{\gamma}^{-1}=-K_{\gamma} \Phi_{*}^{-1} B_{*} \varrho A_{\gamma}^{-1}
$$

holds, where $K_{\gamma}$ and $\Phi_{*}$ are as in (4.6) and (4.9), respectively.

(ii) The formula

$$
A_{*}^{-1}-A_{\nu}^{-1}=-K_{\nu} \Psi_{*}^{-1} B_{*} \varrho A_{\nu}^{-1}
$$

holds, where $K_{\nu}$ and $\Psi_{*}$ are as in (4.8) and (4.9), respectively.

Proof. (i) Recall that, by our assumptions, $A_{*}$ and $A_{\gamma}$ are strictly positive. Let $f \in L_{2}\left(\mathbb{R}^{n}\right)$ and note that $u:=A_{*}^{-1} f$ is the unique solution of the semi-homogeneous boundary value problem

$$
\mathcal{A}_{+} u_{+} \oplus \mathcal{A}_{-} u_{-}=f, \quad B_{*} \varrho u=0,
$$

in $H^{2}\left(\Omega_{+}\right) \oplus H^{2}\left(\Omega_{-}\right)$. Define the functions

$$
v:=A_{\gamma}^{-1} f \quad \text { and } \quad z:=u-v .
$$


The latter satisfies

$$
\mathcal{A}_{+} z_{+} \oplus \mathcal{A}_{-} z_{-}=0, \quad B_{*} \varrho z=-B_{*} \varrho v,
$$

which, by (4.7), implies that

$$
z=K_{\gamma} B_{\gamma} \varrho z .
$$

Now it follows from (4.14), (4.9), (4.13) and (4.12) that

$$
\begin{aligned}
z & =K_{\gamma} B_{\gamma} \varrho z=K_{\gamma} \Phi_{*}^{-1} B_{*} \varrho K_{\gamma} B_{\gamma} \varrho z=K_{\gamma} \Phi_{*}^{-1} B_{*} \varrho z \\
& =-K_{\gamma} \Phi_{*}^{-1} B_{*} \varrho v=-K_{\gamma} \Phi_{*}^{-1} B_{*} \varrho A_{\gamma}^{-1} f .
\end{aligned}
$$

This, together with $A_{*}^{-1} f=u=v+z$ yields the formula in (i).

Item (ii) can be proved in the same way as item (i) when $A_{\gamma}, B_{\gamma}, K_{\gamma}$ and $\Phi_{*}$ are replaced by $A_{\nu}, B_{\nu}, K_{\nu}$ and $\Psi_{*}$, respectively.

In the next proposition we simplify the formula from Theorem 4.3 (i) for the difference between the inverses of the self-adjoint operators $A_{\delta, \alpha}$ and $A_{\gamma}$. In the formulation and the proof of this proposition we employ the column operator

$$
\widetilde{K}_{\gamma}:=\left(\begin{array}{l}
K_{\gamma}^{+} \\
K_{\gamma}^{-}
\end{array}\right): H^{3 / 2}(\Sigma) \rightarrow H^{2}\left(\Omega_{+}\right) \times H^{2}\left(\Omega_{-}\right) .
$$

Proposition 4.4. Let $A_{\delta, \alpha}$ and $A_{\gamma}$ be as above, and let $P_{\gamma, \nu}^{ \pm}$be the Dirichletto-Neumann maps in (3.9) with principal symbols $p_{\gamma, \nu}^{ \pm 0}$ in (3.12). Then the following statements hold.

(i) The $\psi$ do $P_{\gamma, \nu}^{+}+P_{\gamma, \nu}^{-}-\alpha$ is elliptic of order 1 with principal symbol $p_{\gamma, \nu}^{+0}+p_{\gamma, \nu}^{-0}=2 \kappa_{0}$, and it maps $H^{3 / 2}(\Sigma)$ bijectively onto $H^{1 / 2}(\Sigma)$.

(ii) The formula

$$
A_{\delta, \alpha}^{-1}-A_{\gamma}^{-1}=\widetilde{K}_{\gamma}\left(P_{\gamma, \nu}^{+}+P_{\gamma, \nu}^{-}-\alpha\right)^{-1} \widetilde{K}_{\gamma}^{*}
$$

holds, where $\widetilde{K}_{\gamma}$ is as in (4.15) and $\widetilde{K}_{\gamma}^{*}$ is the $L_{2}$-adjoint of $\widetilde{K}_{\gamma}$.

Proof. (i) Both operators $P_{\gamma, \nu}^{+}$and $P_{\gamma, \nu}^{-}$are symmetric first-order elliptic $\psi$ do's on $\Sigma$ (see Lemmas 3.2 (ii) and 3.3) with principal symbols $p_{\gamma, \nu}^{ \pm 0}$ as in (3.12). Hence the $\psi$ do

$$
P_{\gamma, \nu}^{+}+P_{\gamma, \nu}^{-}-\alpha
$$

is also symmetric of order 1 with principal symbol $p_{\gamma, \nu}^{+0}+p_{\gamma, \nu}^{-0}=2 \kappa_{0}$. According to Lemma 3.2 (ii) we have $p_{\gamma, \nu}^{ \pm 0}>0$ for $\xi^{\prime} \neq 0$, and hence (4.16) is elliptic. By [31, Theorem 8.11] the index of the $\psi$ do (4.16) as a mapping from $H^{3 / 2}(\Sigma)$ to $H^{1 / 2}(\Sigma)$ is 0 . Hence, in order to prove bijectivity of (4.16) from $H^{3 / 2}(\Sigma)$ onto $H^{1 / 2}(\Sigma)$, it remains to show that $\operatorname{ker}\left(P_{\gamma, \nu}^{+}+P_{\gamma, \nu}^{-}-\alpha\right)$ is trivial. Suppose for a moment that this were not the case. Then, by [31, Theorem 8.11], there exists a non-trivial $\varphi \in C^{\infty}(\Sigma)$ such that

$$
\left(P_{\gamma, \nu}^{+}+P_{\gamma, \nu}^{-}-\alpha\right) \varphi=0 .
$$


Let us consider the non-trivial function $u:=\widetilde{K}_{\gamma} \varphi$. Then we have $u \in$ $H^{2}\left(\Omega_{+}\right) \oplus H^{2}\left(\Omega_{-}\right), \mathcal{A}_{+} u_{+} \oplus \mathcal{A}_{-} u_{-}=0$, and

$$
\begin{aligned}
\gamma^{+} u_{+}-\gamma^{-} u_{-} & =0, \\
\nu^{+} u_{+}+\nu^{-} u_{-}-\alpha \gamma u & =\nu^{+} K_{\gamma}^{+} \varphi+\nu^{-} K_{\gamma}^{-} \varphi-\alpha \varphi \\
& =P_{\gamma, \nu}^{+} \varphi+P_{\gamma, \nu}^{-} \varphi-\alpha \varphi=0 .
\end{aligned}
$$

From (2.5) we conclude that $u \in \operatorname{dom} A_{\delta, \alpha}$ and, moreover, $A_{\delta, \alpha} u=0$, which contradicts the invertibility of $A_{\delta, \alpha}$.

(ii) Let us consider the matrix $\psi$ do $\Phi_{\delta, \alpha}=B_{\delta, \alpha} \varrho K_{\gamma}$ (cf. (4.9)). For $\left(\begin{array}{c}\varphi_{+} \\ \varphi_{-}\end{array}\right) \in H^{3 / 2}(\Sigma) \times H^{3 / 2}(\Sigma)$ we have

$$
\begin{aligned}
\Phi_{\delta, \alpha}\left(\begin{array}{c}
\varphi_{+} \\
\varphi_{-}
\end{array}\right) & =\left(\begin{array}{cccc}
1 & -1 & 0 & 0 \\
-\alpha & 0 & 1 & 1
\end{array}\right) \varrho K_{\gamma}\left(\begin{array}{c}
\varphi_{+} \\
\varphi_{-}
\end{array}\right) \\
& =\left(\begin{array}{cccc}
1 & -1 & 0 & 0 \\
-\alpha & 0 & 1 & 1
\end{array}\right)\left(\begin{array}{c}
\varphi_{+} \\
\varphi_{-} \\
P_{\gamma, \nu}^{+} \varphi_{+} \\
P_{\gamma, \nu}^{-} \varphi_{-}
\end{array}\right) \\
& =\left(\begin{array}{c}
\varphi_{+}^{-} \varphi_{-} \\
-\alpha \varphi_{+}+P_{\gamma, \nu}^{+} \varphi_{+}+P_{\gamma, \nu}^{-} \varphi_{-}
\end{array}\right),
\end{aligned}
$$

and hence $\Phi_{\delta, \alpha}$ can be written in matrix form as

$$
\Phi_{\delta, \alpha}=\left(\begin{array}{cc}
1 & -1 \\
-\alpha+P_{\gamma, \nu}^{+} & P_{\gamma, \nu}^{-}
\end{array}\right) .
$$

By item (i) the operator $P_{\gamma, \nu}^{+}+P_{\gamma, \nu}^{-}-\alpha$ is bijective from $H^{3 / 2}(\Sigma)$ onto $H^{1 / 2}(\Sigma)$. It follows that the matrix operator

$$
\left(P_{\gamma, \nu}^{+}+P_{\gamma, \nu}^{-}-\alpha\right)^{-1}\left(\begin{array}{cc}
P_{\gamma, \nu}^{-} & 1 \\
\alpha-P_{\gamma, \nu}^{+} & 1
\end{array}\right)
$$

is well defined as a mapping from $H^{3 / 2}(\Sigma) \times H^{1 / 2}(\Sigma)$ into $H^{3 / 2}(\Sigma) \times H^{3 / 2}(\Sigma)$ and that it is the inverse of $\Phi_{\delta, \alpha}$. Indeed, we have

$$
\begin{aligned}
& \left(P_{\gamma, \nu}^{+}+P_{\gamma, \nu}^{-}-\alpha\right)^{-1}\left(\begin{array}{cc}
P_{\gamma, \nu}^{-} & 1 \\
\alpha-P_{\gamma, \nu}^{+} & 1
\end{array}\right)\left(\begin{array}{cc}
1 & -1 \\
-\alpha+P_{\gamma, \nu}^{+} & P_{\gamma, \nu}^{-}
\end{array}\right) \\
& =\left(P_{\gamma, \nu}^{+}+P_{\gamma, \nu}^{-}-\alpha\right)^{-1}\left(\begin{array}{cc}
P_{\gamma, \nu}^{+}+P_{\gamma, \nu}^{-}-\alpha & 0 \\
0 & P_{\gamma, \nu}^{+}+P_{\gamma, \nu}^{-}-\alpha
\end{array}\right)=\left(\begin{array}{ll}
I & 0 \\
0 & I
\end{array}\right)
\end{aligned}
$$

on $H^{3 / 2}(\Sigma) \times H^{3 / 2}(\Sigma)$.

For $f=f_{+} \oplus f_{-} \in L_{2}\left(\mathbb{R}^{n}\right)$ we obtain from Theorem 4.3, (3.15), (4.17) and (4.4) that

$$
\begin{aligned}
A_{\delta, \alpha}^{-1} f-A_{\gamma}^{-1} f & =-\left(\begin{array}{cc}
K_{\gamma}^{+} & 0 \\
0 & K_{\gamma}^{-}
\end{array}\right) \Phi_{\delta, \alpha}^{-1} B_{\delta, \alpha} \varrho A_{\gamma}^{-1} f \\
& =-\left(\begin{array}{cc}
K_{\gamma}^{+} & 0 \\
0 & K_{\gamma}^{-}
\end{array}\right) \Phi_{\delta, \alpha}^{-1} B_{\delta, \alpha}\left(\begin{array}{c}
0 \\
0 \\
\nu^{+} A_{+, \gamma}^{-1} f_{+} \\
\nu^{-} A_{-, \gamma}^{-1} f_{-}
\end{array}\right)
\end{aligned}
$$




$$
\begin{aligned}
& =\left(\begin{array}{cc}
K_{\gamma}^{+} & 0 \\
0 & K_{\gamma}^{-}
\end{array}\right) \Phi_{\delta, \alpha}^{-1}\left(\begin{array}{cccc}
1 & -1 & 0 & 0 \\
-\alpha & 0 & 1 & 1
\end{array}\right)\left(\begin{array}{c}
0 \\
0 \\
\left(K_{\gamma}^{+}\right)^{*} f_{+} \\
\left(K_{\gamma}^{-}\right)^{*} f_{-}
\end{array}\right) \\
& =\left(\begin{array}{cc}
K_{\gamma}^{+} & 0 \\
0 & K_{\gamma}^{-}
\end{array}\right) \Phi_{\delta, \alpha}^{-1}\left(\begin{array}{c}
0 \\
\widetilde{K}_{\gamma}^{*} f
\end{array}\right) \\
& =\left(\begin{array}{l}
K_{\gamma}^{+}\left(P_{\gamma, \nu}^{+}+P_{\gamma, \nu}^{-}-\alpha\right)^{-1} \widetilde{K}_{\gamma}^{*} f \\
K_{\gamma}^{-}\left(P_{\gamma, \nu}^{+}+P_{\gamma, \nu}^{-}-\alpha\right)^{-1} \widetilde{K}_{\gamma}^{*} f
\end{array}\right) \\
& =\widetilde{K}_{\gamma}\left(P_{\gamma, \nu}^{+}+P_{\gamma, \nu}^{-}-\alpha\right)^{-1} \widetilde{K}_{\gamma}^{*} f,
\end{aligned}
$$

which proves item (ii).

Note that the operator $A_{\delta, \alpha}$ with $\alpha \equiv 0$ coincides with the operator $A_{0}$; cf. Section 2. This observation, together with Proposition 4.4 and the relation

$$
\begin{aligned}
& \left(P_{\gamma, \nu}^{+}+P_{\gamma, \nu}^{-}-\alpha\right)^{-1}-\left(P_{\gamma, \nu}^{+}+P_{\gamma, \nu}^{-}\right)^{-1} \\
& \quad=\left(P_{\gamma, \nu}^{+}+P_{\gamma, \nu}^{-}-\alpha\right)^{-1} \alpha\left(P_{\gamma, \nu}^{+}+P_{\gamma, \nu}^{-}\right)^{-1}
\end{aligned}
$$

yields the following corollary.

Corollary 4.5. Under the assumptions of Proposition 4.4 and with $A_{0}$ as in $(2.3)$,

$$
A_{\delta, \alpha}^{-1}-A_{0}^{-1}=\widetilde{K}_{\gamma}\left(P_{\gamma, \nu}^{+}+P_{\gamma, \nu}^{-}-\alpha\right)^{-1} \alpha\left(P_{\gamma, \nu}^{+}+P_{\gamma, \nu}^{-}\right)^{-1} \widetilde{K}_{\gamma}^{*}
$$

holds.

In the next proposition we simplify the formula from Theorem 4.3 (ii) for the difference between the inverses of the self-adjoint operators $A_{\delta^{\prime}, \beta}$ and $A_{\nu}$. The proof follows the same strategy as the proof of Proposition 4.4. For the convenience of the reader we provide the essential arguments. We also mention that the formula in item (ii) below is similar to the one in $[7$, Theorem 3.11 (ii)]. Here we employ the column operator

$$
\widetilde{K}_{\nu}:=\left(\begin{array}{c}
K_{\nu}^{+} \\
-K_{\nu}^{-}
\end{array}\right): H^{1 / 2}(\Sigma) \rightarrow H^{2}\left(\Omega_{+}\right) \times H^{2}\left(\Omega_{-}\right) .
$$

Proposition 4.6. Let $A_{\nu}$ and $A_{\delta^{\prime}, \beta}$ be as defined above, and let $P_{\nu, \gamma}^{ \pm}$be the Neumann-to-Dirichlet maps in (3.9). Then the following statements hold.

(i) The $\psi$ do $\beta-\left(P_{\nu, \gamma}^{+}+P_{\nu, \gamma}^{-}\right)$is elliptic of order 0 with principal symbol $\beta$, and it maps $H^{1 / 2}(\Sigma)$ bijectively onto $H^{1 / 2}(\Sigma)$.

(ii) The formula

$$
A_{\delta^{\prime}, \beta}^{-1}-A_{\nu}^{-1}=\widetilde{K}_{\nu}\left(\beta-\left(P_{\nu, \gamma}^{+}+P_{\nu, \gamma}^{-}\right)\right)^{-1} \widetilde{K}_{\nu}^{*}
$$

holds, where $\widetilde{K}_{\nu}$ is as in (4.19) and $\widetilde{K}_{\nu}^{*}$ is the $L_{2}$-adjoint of $\widetilde{K}_{\nu}$.

Proof. (i) The operators $P_{\nu, \gamma}^{ \pm}$are symmetric elliptic $\psi$ do's on $\Sigma$ of order -1 (see Lemmas 3.2 (ii) and 3.3). Since $\beta$ is real-valued and non-zero on $\Sigma$, $\beta-\left(P_{\nu, \gamma}^{+}+P_{\nu, \gamma}^{-}\right)$is a symmetric and elliptic $\psi$ do of order 0 with principal symbol $\beta$. Hence, by [31, Theorem 8.11], its index as a mapping from $H^{1 / 2}(\Sigma)$ into $H^{1 / 2}(\Sigma)$ is 0 . Therefore it suffices to verify the injectivity of 
$\beta-\left(P_{\nu, \gamma}^{+}+P_{\nu, \gamma}^{-}\right)$. Suppose that this were not the case. As in the proof of Proposition 4.4 (i) it follows that there exists a non-trivial $\psi \in C^{\infty}(\Sigma)$ such that $\left(\beta-\left(P_{\nu, \gamma}^{+}+P_{\nu, \gamma}^{-}\right)\right) \psi=0$ and the function $\widetilde{K}_{\nu} \psi \neq 0$ belongs to ker $A_{\delta^{\prime}, \beta}$; this is a contradiction to the invertibility of $A_{\delta^{\prime}, \beta}$.

(ii) A simple calculation shows that the matrix $\psi$ do $\Psi_{\delta^{\prime}, \beta}=B_{\delta^{\prime}, \beta} \varrho K_{\nu}$ acts as

$$
\Psi_{\delta^{\prime}, \beta}\left(\begin{array}{l}
\psi_{+} \\
\psi_{-}
\end{array}\right)=\left(\begin{array}{cccc}
1 & -1 & -\beta & 0 \\
0 & 0 & 1 & 1
\end{array}\right) \varrho K_{\nu}\left(\begin{array}{l}
\psi_{+} \\
\psi_{-}
\end{array}\right)=\left(\begin{array}{cc}
P_{\nu, \gamma}^{+}-\beta & -P_{\nu, \gamma}^{-} \\
1 & 1
\end{array}\right)\left(\begin{array}{l}
\psi_{+} \\
\psi_{-}
\end{array}\right),
$$

and a similar consideration as in the proof of Proposition 4.4 (ii) yields

$$
\Psi_{\delta^{\prime}, \beta}^{-1}=\left(P_{\nu, \gamma}^{+}+P_{\nu, \gamma}^{-}-\beta\right)^{-1}\left(\begin{array}{cc}
1 & P_{\nu, \gamma}^{-} \\
-1 & P_{\nu, \gamma}^{+}-\beta
\end{array}\right) .
$$

It is seen from the form of $B_{\delta^{\prime}, \beta}$ in (4.4), relations (3.15), (4.20) and Theorem 4.3 (ii) that

$$
\begin{aligned}
& A_{\delta^{\prime}, \beta}^{-1} f-A_{\nu}^{-1} f=-\left(\begin{array}{cc}
K_{\nu}^{+} & 0 \\
0 & K_{\nu}^{-}
\end{array}\right) \Psi_{\delta^{\prime}, \beta}^{-1} B_{\delta^{\prime}, \beta}\left(\begin{array}{c}
\left(K_{\nu}^{+}\right)^{*} f_{+} \\
\left(K_{\nu}^{-}\right)^{*} f_{-} \\
0 \\
0
\end{array}\right) \\
& =-\left(\begin{array}{cc}
K_{\nu}^{+} & 0 \\
0 & K_{\nu}^{-}
\end{array}\right)\left(P_{\nu, \gamma}^{+}+P_{\nu, \gamma}^{-}-\beta\right)^{-1}\left(\begin{array}{c}
\left(K_{\nu}^{+}\right)^{*} f_{+}-\left(K_{\nu}^{-}\right)^{*} f_{-} \\
\left(K_{\nu}^{-}\right)^{*} f_{-}-\left(K_{\nu}^{+}\right)^{*} f_{+}
\end{array}\right) \\
& =\widetilde{K}_{\nu}\left(\beta-\left(P_{\nu, \gamma}^{+}+P_{\nu, \gamma}^{-}\right)\right)^{-1} \widetilde{K}_{\nu}^{*} f
\end{aligned}
$$

holds for all $f \in L_{2}\left(\mathbb{R}^{n}\right)$, which proves (ii).

Finally we provide a more explicit formula for the difference between the inverses of the self-adjoint operators $A_{0}$ and $A_{\nu}$. Again the proof follows the same strategy as the proofs of Propositions 4.4 and 4.6.

Proposition 4.7. Let $A_{0}$ and $A_{\nu}$ be as above, and let $P_{\nu, \gamma}^{ \pm}$be the Neumannto-Dirichlet maps $P_{\nu, \gamma}^{ \pm}$in (3.9) with principal symbols $p_{\nu, \gamma}^{ \pm 0}$ in (3.12). Then the following statements hold.

(i) The $\psi$ do $P_{\nu, \gamma}^{+}+P_{\nu, \gamma}^{-}$is elliptic of order -1 with principal symbol $p_{\nu, \gamma}^{+0}+$ $p_{\nu, \gamma}^{-0}$, and it maps $H^{1 / 2}(\Sigma)$ bijectively onto $H^{3 / 2}(\Sigma)$.

(ii) The formula

$$
A_{\nu}^{-1}-A_{0}^{-1}=\widetilde{K}_{\nu}\left(P_{\nu, \gamma}^{+}+P_{\nu, \gamma}^{-}\right)^{-1} \widetilde{K}_{\nu}^{*}
$$

holds, where $\widetilde{K}_{\nu}$ is as in (4.19).

Proof. (i) Following the arguments in the proofs of Proposition 4.4 and Proposition 4.6 we conclude that the $\psi$ do $P_{\nu, \gamma}^{+}+P_{\nu, \gamma}^{-}$is elliptic of order -1 with principal symbol $p_{\nu, \gamma}^{+0}+p_{\nu, \gamma}^{-0}$, and its index as a mapping from $H^{1 / 2}(\Sigma)$ into $H^{3 / 2}(\Sigma)$ is 0 . Again it is sufficient for the bijectivity to verify that $\operatorname{ker}\left(P_{\nu, \gamma}^{+}+P_{\nu, \gamma}^{-}\right)$is trivial. Suppose that this were not the case. Then it follows that there exists a non-trivial $\psi \in C^{\infty}(\Sigma)$ such that $\left(P_{\nu, \gamma}^{+}+P_{\nu, \gamma}^{-}\right) \psi=0$ and the function $\widetilde{K}_{\nu} \psi \neq 0$ belongs to ker $A_{0}$, a contradiction to the invertibility of $A_{0}$. 
(ii) The matrix $\psi$ do $\Psi_{0}=B_{0} \varrho K_{\nu}$ and its inverse have the form

$$
\Psi_{0}=\left(\begin{array}{cc}
P_{\nu, \gamma}^{+} & -P_{\nu, \gamma}^{-} \\
1 & 1
\end{array}\right) \quad \text { and } \Psi_{0}^{-1}=\left(P_{\nu, \gamma}^{+}+P_{\nu, \gamma}^{-}\right)^{-1}\left(\begin{array}{cc}
1 & P_{\nu, \gamma}^{-} \\
-1 & P_{\nu, \gamma}^{+}
\end{array}\right) \text {. }
$$

Hence it follows from (3.15), the form of $B_{0}$ in (4.4) and Theorem 4.3 (ii) that

$$
\begin{aligned}
A_{\nu}^{-1} f-A_{0}^{-1} f & =\left(\begin{array}{cc}
K_{\nu}^{+} & 0 \\
0 & K_{\nu}^{-}
\end{array}\right) \Psi_{0}^{-1} B_{0}\left(\begin{array}{c}
\left(K_{\nu}^{+}\right)^{*} f_{+} \\
\left(K_{\nu}^{-}\right)^{*} f_{-} \\
0 \\
0
\end{array}\right) \\
& =\left(\begin{array}{cc}
K_{\nu}^{+} & 0 \\
0 & K_{\nu}^{-}
\end{array}\right)\left(P_{\nu, \gamma}^{+}+P_{\nu, \gamma}^{-}\right)^{-1}\left(\begin{array}{c}
\left(K_{\nu}^{+}\right)^{*} f_{+}-\left(K_{\nu}^{-}\right)^{*} f_{-} \\
\left(K_{\nu}^{-}\right)^{*} f_{-}-\left(K_{\nu}^{+}\right)^{*} f_{+}
\end{array}\right) \\
& =\widetilde{K}_{\nu}\left(P_{\nu, \gamma}^{+}+P_{\nu, \gamma}^{-}\right)^{-1} \widetilde{K}_{\nu}^{*} f
\end{aligned}
$$

holds for all $f \in L_{2}\left(\mathbb{R}^{n}\right)$, which proves (ii).

Corollary 4.8. Under the assumptions of Proposition 4.7 and with $A_{\delta^{\prime}, \beta}$ as in (2.6),

$$
A_{\delta^{\prime}, \beta}^{-1}-A_{0}^{-1}=\widetilde{K}_{\nu}\left(P_{\nu, \gamma}^{+}+P_{\nu, \gamma}^{-}\right)^{-1} \beta\left(\beta-\left(P_{\nu, \gamma}^{+}+P_{\nu, \gamma}^{-}\right)\right)^{-1} \widetilde{K}_{\nu}^{*}
$$

holds.

\section{Spectral asymptotics FOR RESOlVent Differences}

In this section we present and prove the main results of this note, namely, we obtain spectral asymptotics formulae for the differences between the inverses of the operators $A_{0}, A_{\nu}, A_{\delta, \alpha}$ and $A_{\delta^{\prime}, \beta}$ introduced in Section 2. These asymptotics refine some spectral estimates for resolvent differences found in $[6,7]$. The proofs are based on the Krein-type resolvent formulae proved in Section 4, spectral asymptotics for $\psi$ do's on smooth manifolds without boundary and some elements of the $\psi$ dbo calculus.

In all theorems of this section we suppose that the assumptions at the beginning of Section 2 hold. Moreover, let $\underline{a}_{n n}$ and $\kappa_{0}$ be defined as in (3.2) and (3.4). The next theorem contains one of our main results: the spectral asymptotics of the difference between the inverses of $A_{\delta, \alpha}$ and $A_{0}$.

Theorem 5.1. Let $\alpha \in C^{\infty}(\Sigma)$ be real-valued, let $A_{\delta, \alpha}$ be the self-adjoint operator in (2.5) and let $A_{0}$ be the free operator in (2.3). Then

$$
A_{\delta, \alpha}^{-1}-A_{0}^{-1}
$$

is a compact operator in $L_{2}\left(\mathbb{R}^{n}\right)$ and the following two statements hold.

(i) The singular values $s_{k}$ of $A_{\delta, \alpha}^{-1}-A_{0}^{-1}$ satisfy

$$
s_{k}=C_{\delta, \alpha} k^{-\frac{3}{n-1}}+\mathrm{o}\left(k^{-\frac{3}{n-1}}\right), \quad k \rightarrow \infty,
$$

with the constant $C_{\delta, \alpha}=\left(C_{\delta, \alpha}^{\prime}\right)^{\frac{3}{n-1}}$,

$$
C_{\delta, \alpha}^{\prime}=\frac{1}{(n-1)(2 \pi)^{n-1}} \int_{\Sigma} \int_{\left|\xi^{\prime}\right|=1}\left(\frac{\underline{a}_{n n}\left(x^{\prime}\right)\left|\alpha\left(x^{\prime}\right)\right|}{4\left(\kappa_{0}\left(x^{\prime}, \xi^{\prime}\right)\right)^{3}}\right)^{\frac{n-1}{3}} \mathrm{~d} \omega\left(\xi^{\prime}\right) \mathrm{d} \sigma\left(x^{\prime}\right) .
$$


(ii) If $\alpha\left(x^{\prime}\right) \neq 0$ for all $x^{\prime} \in \Sigma$, then the singular values $s_{k}$ of $A_{\delta, \alpha}^{-1}-A_{0}^{-1}$ satisfy

$$
s_{k}=C_{\delta, \alpha} k^{-\frac{3}{n-1}}+\mathrm{O}\left(k^{-\frac{4}{n-1}}\right), \quad k \rightarrow \infty
$$

Proof. (i) Let us set

$$
G_{\delta, \alpha}:=A_{\delta, \alpha}^{-1}-A_{0}^{-1} \quad \text { and } \quad S_{\alpha}:=\left(P_{\gamma, \nu}^{+}+P_{\gamma, \nu}^{-}-\alpha\right)^{-1} \alpha\left(P_{\gamma, \nu}^{+}+P_{\gamma, \nu}^{-}\right)^{-1}
$$

where $P_{\gamma, \nu}^{+}$and $P_{\gamma, \nu}^{-}$are defined in (3.9), and let $\widetilde{K}_{\gamma}$ be as in (4.15). It follows from Corollary 4.5 that

$$
G_{\delta, \alpha}=\widetilde{K}_{\gamma} S_{\alpha} \widetilde{K}_{\gamma}^{*}
$$

which is a bounded self-adjoint operator in $L_{2}\left(\mathbb{R}^{n}\right)$. We also make use of the operator

$$
R_{\gamma}:=\widetilde{K}_{\gamma}^{*} \widetilde{K}_{\gamma}=\left(K_{\gamma}^{+}\right)^{*} K_{\gamma}^{+}+\left(K_{\gamma}^{-}\right)^{*} K_{\gamma}^{-}
$$

which is a $\psi$ do on $\Sigma$ of order -1 with the principal symbol $\underline{a}_{n n} / \kappa_{0}$ according to Lemma 3.2 (iii). Note that for a non-trivial $f \in L_{2}(\Sigma)$ one has

$$
\left(\left(K_{\gamma}^{ \pm}\right)^{*} K_{\gamma}^{ \pm} f, f\right)_{L_{2}(\Sigma)}=\left\|K_{\gamma}^{ \pm} f\right\|_{L_{2}\left(\Omega_{ \pm}\right)}^{2}>0
$$

Thus the non-negative self-adjoint operators $\left(K_{\gamma}^{ \pm}\right)^{*} K_{\gamma}^{ \pm}$are invertible; hence so is $R_{\gamma}$.

In the following we use that in the pseudodifferential boundary operator calculus, $\widetilde{K}_{\gamma}, \widetilde{K}_{\gamma}^{*}$ and $R_{\gamma}$ extend to continuous operators

$$
\begin{array}{ll}
\widetilde{K}_{\gamma}: H^{s}(\Sigma) \rightarrow H^{s+\frac{1}{2}}\left(\Omega_{+}\right) \times H^{s+\frac{1}{2}}\left(\Omega_{-}\right) & \text {for } s \in \mathbb{R}, \\
\widetilde{K}_{\gamma}^{*}: H^{t}\left(\Omega_{+}\right) \times H^{t}\left(\Omega_{-}\right) \rightarrow H^{t+\frac{1}{2}}(\Sigma) & \text { for } t>-\frac{1}{2}, \\
R_{\gamma}: H^{s}(\Sigma) \rightarrow H^{s+1}(\Sigma) & \text { for } s \in \mathbb{R}
\end{array}
$$

(we use the same notation for the extended/restricted operators). By Proposition 4.4 (i) and Lemma 3.2 (ii) the $\psi$ do's $\left(P_{\gamma, \nu}^{+}+P_{\gamma, \nu}^{-}\right)^{-1}$ and $\left(P_{\gamma, \nu}^{+}+P_{\gamma, \nu}^{-}-\right.$ $\alpha)^{-1}$ are both of order -1 and have the principal symbol $1 /\left(2 \kappa_{0}\right)$. Hence $S_{\alpha}$ is of order -2 and has the principal symbol $\alpha /\left(4 \kappa_{0}^{2}\right)$. It extends to a mapping

$$
S_{\alpha}: H^{s}(\Sigma) \rightarrow H^{s+2}(\Sigma) \quad \text { for } s \in \mathbb{R}
$$

It now follows from (5.1) that

$$
\begin{aligned}
G_{\delta, \alpha}^{*} G_{\delta, \alpha} & =\left(\widetilde{K}_{\gamma} S_{\alpha} \widetilde{K}_{\gamma}^{*}\right)^{*} \widetilde{K}_{\gamma} S_{\alpha} \widetilde{K}_{\gamma}^{*} \\
& =\widetilde{K}_{\gamma} S_{\alpha}^{*} \widetilde{K}_{\gamma}^{*} \widetilde{K}_{\gamma} S_{\alpha} \widetilde{K}_{\gamma}^{*} \\
& =\widetilde{K}_{\gamma} S_{\alpha} R_{\gamma} S_{\alpha} \widetilde{K}_{\gamma}^{*}
\end{aligned}
$$


where we used the symmetry of $S_{\alpha}$, which follows from (4.18). Therefore (here $s_{k}(T)$ denotes the $k$ th singular value and $\lambda_{k}(T)$ the $k$ th positive eigenvalue of an operator $T$ )

$$
\begin{aligned}
\left(s_{k}\left(G_{\delta, \alpha}\right)\right)^{2} & =\lambda_{k}\left(G_{\delta, \alpha}^{*} G_{\delta, \alpha}\right) \\
& =\lambda_{k}\left(\widetilde{K}_{\gamma} S_{\alpha} R_{\gamma} S_{\alpha} \widetilde{K}_{\gamma}^{*}\right) \\
& =\lambda_{k}\left(S_{\alpha} R_{\gamma} S_{\alpha} \widetilde{K}_{\gamma}^{*} \widetilde{K}_{\gamma}\right) \\
& =\lambda_{k}\left(S_{\alpha} R_{\gamma} S_{\alpha} R_{\gamma}\right) \\
& =\lambda_{k}\left(R_{\gamma}^{1 / 2} S_{\alpha} R_{\gamma}^{1 / 2} R_{\gamma}^{1 / 2} S_{\alpha} R_{\gamma}^{1 / 2}\right) \\
& =\left(s_{k}\left(R_{\gamma}^{1 / 2} S_{\alpha} R_{\gamma}^{1 / 2}\right)\right)^{2}
\end{aligned}
$$

that is, the singular values $s_{k}$ of $A_{\delta, \alpha}^{-1}-A_{0}^{-1}$ are

$$
s_{k}\left(G_{\delta, \alpha}\right)=s_{k}\left(R_{\gamma}^{1 / 2} S_{\alpha} R_{\gamma}^{1 / 2}\right) .
$$

Here since $R_{\gamma}$ is non-negative and invertible, the operator $R_{\gamma}^{1 / 2}$ is well defined as an elliptic $\psi$ do of order $-1 / 2$ with principal symbol $\left(\underline{a}_{n n} / \kappa_{0}\right)^{1 / 2}$, by [47]. Moreover, $S_{\alpha}$ is a $\psi$ do of order -2 with principal symbol $\alpha /\left(4 \kappa_{0}^{2}\right)$; so standard rules of the $\psi$ do calculus yield that the $\psi$ do

$$
P_{\delta, \alpha}:=R_{\gamma}^{1 / 2} S_{\alpha} R_{\gamma}^{1 / 2}
$$

is of order -3 and with the principal symbol $\left(\underline{a}_{n n} \alpha\right) /\left(4 \kappa_{0}^{3}\right)$. Now Theorem 3.1 (i) implies that

$$
s_{k}\left(P_{\delta, \alpha}\right)=C_{\delta, \alpha} k^{-\frac{3}{n-1}}+\mathrm{o}\left(k^{-\frac{3}{n-1}}\right), \quad k \rightarrow \infty,
$$

with $C_{\delta, \alpha}$ as in the formulation of the theorem.

(ii) Recall that $\underline{a}_{n n}\left(x^{\prime}\right)>0$ and $\kappa_{0}\left(x^{\prime}, \xi^{\prime}\right)>0$ for $\xi^{\prime} \neq 0$; cf. Section 3 . Hence the assumption $\alpha\left(x^{\prime}\right) \neq 0$ for all $x^{\prime} \in \Sigma$ implies that the $\psi$ do $P_{\delta, \alpha}$ in (5.3) is elliptic. Moreover, since $S_{\alpha}$ is then invertible and $R_{\gamma}^{1 / 2}$ is invertible, the operator $P_{\delta, \alpha}$ in (5.3) is invertible. Now Theorem 3.1 (ii) implies the assertion.

The next theorem contains our second main result: the spectral asymptotics of the difference between the inverses of $A_{\delta^{\prime}, \beta}$ and $A_{0}$. It turns out that the principal term in the asymptotics is independent of $\beta$. The proof of Theorem 5.2 is very similar to the proof of Theorem 5.1.

Theorem 5.2. Let $\beta \in C^{\infty}(\Sigma)$ be real-valued such that $\beta\left(x^{\prime}\right) \neq 0$ for all $x^{\prime} \in \Sigma$, let $A_{\delta^{\prime}, \beta}$ be the self-adjoint operator in (2.6) and let $A_{0}$ be the free operator in (2.3). Then

$$
A_{\delta^{\prime}, \beta}^{-1}-A_{0}^{-1}
$$

is a compact operator in $L_{2}\left(\mathbb{R}^{n}\right)$, and its singular values satisfy

$$
s_{k}=C_{\delta^{\prime}} k^{-\frac{2}{n-1}}+\mathrm{O}\left(k^{-\frac{3}{n-1}}\right), \quad k \rightarrow \infty,
$$

with the constant $C_{\delta^{\prime}}=\left(C_{\delta^{\prime}}^{\prime}\right)^{\frac{2}{n-1}}$,

$$
C_{\delta^{\prime}}^{\prime}=\frac{1}{(n-1)(2 \pi)^{n-1}} \int_{\Sigma} \int_{\left|\xi^{\prime}\right|=1}\left(\frac{\underline{a}_{n n}\left(x^{\prime}\right)}{2\left(\kappa_{0}\left(x^{\prime}, \xi^{\prime}\right)\right)^{2}}\right)^{\frac{n-1}{2}} \mathrm{~d} \omega\left(\xi^{\prime}\right) \mathrm{d} \sigma\left(x^{\prime}\right) .
$$


Proof. According to Corollary 4.8 we have

$$
A_{\delta^{\prime}, \beta}^{-1}-A_{0}^{-1}=\widetilde{K}_{\nu}\left(P_{\nu, \gamma}^{+}+P_{\nu, \gamma}^{-}\right)^{-1} \beta\left(\beta-\left(P_{\nu, \gamma}^{+}+P_{\nu, \gamma}^{-}\right)\right)^{-1} \widetilde{K}_{\nu}^{*}
$$

where $P_{\nu, \gamma}^{+}, P_{\nu, \gamma}^{-}$and $\widetilde{K}_{\nu}$ are as in (3.9) and (4.19), respectively. Let us set

$$
R_{\nu}:=\widetilde{K}_{\nu}^{*} \widetilde{K}_{\nu} \quad \text { and } \quad T_{\beta}:=\left(P_{\nu, \gamma}^{+}+P_{\nu, \gamma}^{-}\right)^{-1} \beta\left(\beta-\left(P_{\nu, \gamma}^{+}+P_{\nu, \gamma}^{-}\right)\right)^{-1} .
$$

Since $K_{\nu}^{ \pm}$are injective, the non-negative self-adjoint operators $\left(K_{\nu}^{ \pm}\right)^{*} K_{\nu}^{ \pm}$ are invertible; hence so is $R_{\nu}$. As in the proof of Theorem 5.1 we make use of the fact that $\widetilde{K}_{\nu}, \widetilde{K}_{\nu}^{*}$ and $T_{\beta}$ extend to continuous operators between the respective spaces. Then the same computation as in (5.2) implies

$$
s_{k}\left(A_{\delta^{\prime}, \beta}^{-1}-A_{0}^{-1}\right)=s_{k}\left(R_{\nu}^{1 / 2} T_{\beta} R_{\nu}^{1 / 2}\right) .
$$

The operator $R_{\nu}^{1 / 2}$ is well defined as an elliptic $\psi$ do of order $-3 / 2$ with principal symbol $\left(\underline{a}_{n n} / \kappa_{0}^{3}\right)^{1 / 2}$ by [47]. It follows from Lemma 3.2 (ii) and Proposition 4.6 (i) that $T_{\beta}$ is a $\psi$ do of order 1 with principal symbol $\kappa_{0} / 2$. By standard rules of the calculus the $\psi$ do

$$
P_{\delta^{\prime}, \beta}:=R_{\nu}^{1 / 2} T_{\beta} R_{\nu}^{1 / 2}
$$

is of order -2 and has the principal symbol $\underline{a}_{n n} /\left(2 \kappa_{0}^{2}\right)$. Since $\underline{a}_{n n}\left(x^{\prime}\right)>0$ and $\kappa_{0}\left(x^{\prime}, \xi^{\prime}\right)>0$ for $\xi^{\prime} \neq 0$, it follows that the operator $P_{\delta^{\prime}, \beta}$ is elliptic. Since $T_{\beta}$ and $R_{\nu}^{1 / 2}$ are invertible the same true for $P_{\delta^{\prime}, \beta}$ in (5.4). Now Theorem 3.1 (ii) yields the spectral asymptotics

$$
s_{k}\left(P_{\delta^{\prime}, \beta}\right)=C_{\delta^{\prime}} k^{-\frac{2}{n-1}}+\mathrm{O}\left(k^{-\frac{3}{n-1}}\right), \quad k \rightarrow \infty,
$$

with $C_{\delta^{\prime}}$ as in the formulation of the theorem.

Finally we state a third result on spectral asymptotics. In the next theorem the difference of the inverses of $A_{\delta^{\prime}, \beta}$ and the orthogonal sum of the Neumann operators $A_{\nu}$ is considered. The proof is similar to the proofs of the previous theorems. Therefore we just give the main arguments.

Theorem 5.3. Let $\beta \in C^{\infty}(\Sigma)$ be real-valued such that $\beta\left(x^{\prime}\right) \neq 0$ for all $x^{\prime} \in \Sigma$, let $A_{\delta^{\prime}, \beta}$ be the self-adjoint operator in (2.6) and let $A_{\nu}$ be as in (2.4). Then

$$
A_{\delta^{\prime}, \beta}^{-1}-A_{\nu}^{-1}
$$

is a compact operator in $L_{2}\left(\mathbb{R}^{n}\right)$, and its singular values satisfy

$$
s_{k}=C_{\delta^{\prime}, \beta, \nu} k^{-\frac{3}{n-1}}+\mathrm{O}\left(k^{-\frac{4}{n-1}}\right), \quad k \rightarrow \infty,
$$

with the constant $C_{\delta^{\prime}, \beta, \nu}=\left(C_{\delta^{\prime}, \beta, \nu}^{\prime}\right)^{\frac{3}{n-1}}$,

$$
C_{\delta^{\prime}, \beta, \nu}^{\prime}=\frac{1}{(n-1)(2 \pi)^{n-1}} \int_{\Sigma} \int_{\left|\xi^{\prime}\right|=1}\left(\frac{\underline{a}_{n n}\left(x^{\prime}\right)}{\left|\beta\left(x^{\prime}\right)\right|\left(\kappa_{0}\left(x^{\prime}, \xi^{\prime}\right)\right)^{3}}\right)^{\frac{n-1}{3}} \mathrm{~d} \omega\left(\xi^{\prime}\right) \mathrm{d} \sigma\left(x^{\prime}\right) .
$$

Proof. According to Proposition 4.6 (ii) we have

$$
A_{\delta^{\prime}, \beta}^{-1}-A_{\nu}^{-1}=\widetilde{K}_{\nu}\left(\beta-\left(P_{\nu, \gamma}^{+}+P_{\nu, \gamma}^{-}\right)\right)^{-1} \widetilde{K}_{\nu}^{*},
$$

where $P_{\nu, \gamma}^{+}, P_{\nu, \gamma}^{-}$and $\widetilde{K}_{\nu}$ are as in (3.9) and (4.19), respectively. Let

$$
R_{\nu}:=\widetilde{K}_{\nu}^{*} \widetilde{K}_{\nu} \quad \text { and } \quad S_{\beta}:=\left(\beta-\left(P_{\nu, \gamma}^{+}+P_{\nu, \gamma}^{-}\right)\right)^{-1},
$$


and observe that $\widetilde{K}_{\nu}, \widetilde{K}_{\nu}^{*}$ and $S_{\beta}$ extend to continuous operators between the respective spaces. As in the proofs of Theorems 5.1 and 5.2 one verifies

$$
s_{k}\left(A_{\delta^{\prime}, \beta}^{-1}-A_{\nu}^{-1}\right)=s_{k}\left(R_{\nu}^{1 / 2} S_{\beta} R_{\nu}^{1 / 2}\right) .
$$

Furthermore, $R_{\nu}^{1 / 2}$ is a $\psi$ do of order $-3 / 2$ with principal symbol $\left(\underline{a}_{n n} / \kappa_{0}^{3}\right)^{1 / 2}$, and by Proposition 4.6 (i) the $\psi$ do $S_{\beta}$ is of order 0 with principal symbol $\beta^{-1}$. Therefore the $\psi$ do

$$
P_{\delta^{\prime}, \beta, \nu}:=R_{\nu}^{1 / 2} S_{\beta} R_{\nu}^{1 / 2}
$$

is of order -3 and has the principal symbol $\underline{a}_{n n} /\left(\beta \kappa_{0}^{3}\right)$. It also follows that $P_{\delta^{\prime}, \beta, \nu}$ is elliptic and invertible, and hence Theorem 3.1 (ii) yields the spectral asymptotics

$$
s_{k}\left(P_{\delta^{\prime}, \beta, \nu}\right)=C_{\delta^{\prime}, \beta, \nu} k^{-\frac{3}{n-1}}+\mathrm{O}\left(k^{-\frac{4}{n-1}}\right), \quad k \rightarrow \infty,
$$

with $C_{\delta^{\prime}, \beta, \nu}$ of the form as stated in the theorem.

The constants $C_{\delta, \alpha}^{\prime}, C_{\delta^{\prime}}^{\prime}$ and $C_{\delta^{\prime}, \beta, \nu}^{\prime}$ in the previous theorems can be computed explicitly in the special case $\mathcal{A}=-\Delta$.

Example 5.4. Denote by $|\Sigma|$ the area of $\Sigma$ and by $\left|S_{n-1}\right|$ the area of the $(n-1)$-dimensional unit sphere (for $n=2$ we have $\left|S_{n-1}\right|=2$ ). According to Example 3.4 in the special case $\mathcal{A}=-\Delta$ the constant $C_{\delta, \alpha}^{\prime}$ in Theorem 5.1 is

$$
\begin{aligned}
C_{\delta, \alpha}^{\prime} & =\frac{1}{(n-1)(2 \pi)^{n-1}} \int_{\Sigma} \int_{\left|\xi^{\prime}\right|=1}\left(\frac{1 \cdot\left|\alpha\left(x^{\prime}\right)\right|}{4\left|\xi^{\prime}\right|^{3}}\right)^{\frac{n-1}{3}} \mathrm{~d} \omega\left(\xi^{\prime}\right) \mathrm{d} \sigma\left(x^{\prime}\right) \\
& =\frac{\left|S_{n-1}\right|}{(n-1)(2 \pi)^{n-1} 4^{(n-1) / 3}} \int_{\Sigma}\left|\alpha\left(x^{\prime}\right)\right|^{\frac{n-1}{3}} \mathrm{~d} \sigma\left(x^{\prime}\right),
\end{aligned}
$$

the constant $C_{\delta^{\prime}}^{\prime}$ in Theorem 5.2 is

$$
\begin{aligned}
C_{\delta^{\prime}}^{\prime} & =\frac{1}{(n-1)(2 \pi)^{n-1}} \int_{\Sigma} \int_{\left|\xi^{\prime}\right|=1}\left(\frac{1}{2\left|\xi^{\prime}\right|^{2}}\right)^{\frac{n-1}{2}} \mathrm{~d} \omega\left(\xi^{\prime}\right) \mathrm{d} \sigma\left(x^{\prime}\right) \\
& =\frac{|\Sigma| \cdot\left|S_{n-1}\right|}{(n-1)(2 \pi)^{n-1} 2^{(n-1) / 2}},
\end{aligned}
$$

and the constant $C_{\delta^{\prime}, \beta, \nu}^{\prime}$ in Theorem 5.3 is

$$
\begin{aligned}
C_{\delta^{\prime}, \beta, \nu}^{\prime} & =\frac{1}{(n-1)(2 \pi)^{n-1}} \int_{\Sigma} \int_{\left|\xi^{\prime}\right|=1}\left(\frac{1}{\left|\beta\left(x^{\prime}\right)\right| \cdot\left|\xi^{\prime}\right|^{3}}\right)^{\frac{n-1}{3}} \mathrm{~d} \omega\left(\xi^{\prime}\right) \mathrm{d} \sigma\left(x^{\prime}\right) \\
& =\frac{\left|S_{n-1}\right|}{(n-1)(2 \pi)^{n-1}} \int_{\Sigma}\left(\frac{1}{\left|\beta\left(x^{\prime}\right)\right|}\right)^{\frac{n-1}{3}} \mathrm{~d} \sigma\left(x^{\prime}\right) .
\end{aligned}
$$

Remark 5.5. We note that the Krein-type formulae in Section 4 for the differences of the inverses $A_{\delta, \alpha}^{-1}-A_{0}^{-1}, A_{\delta^{\prime}, \beta}^{-1}-A_{0}^{-1}$ and $A_{\delta^{\prime}, \beta}^{-1}-A_{\nu}^{-1}$ can be generalized for the differences of the resolvents $\left(A_{\delta, \alpha}-\lambda\right)^{-1}-\left(A_{0}-\lambda\right)^{-1}$, $\left(A_{\delta^{\prime}, \beta}-\lambda\right)^{-1}-\left(A_{0}-\lambda\right)^{-1}$ and $\left(A_{\delta^{\prime}, \beta}-\lambda\right)^{-1}-\left(A_{\nu}-\lambda\right)^{-1}$ for all $\lambda$ in the respective resolvent sets. Making use of such resolvent formulae one 
can show that the spectral asymptotics in Theorem 5.1, Theorem 5.2 and Theorem 5.3 remain true for all $\lambda$ in the respective resolvent sets.

\section{REFERENCES}

[1] D. Alpay and J. Behrndt, Generalized $Q$-functions and Dirichlet-to-Neumann maps for elliptic differential operators, J. Funct. Anal. 257 (2009), 1666-1694.

[2] J.-P. Antoine, F. Gesztesy and J. Shabani, Exactly solvable models of sphere interactions in quantum mechanics, J. Phys. A 20 (1987), 3687-3712.

[3] C. Bardos, J. C. Guillot and J. Ralston, La relation de Poisson pour l'équation des ondes dans un ouvert non borné. Application à la théorie de la diffusion, Comm. Partial Differential Equations 7 (1982), 905-958.

[4] J. Behrndt, P. Exner and V. Lotoreichik, Schrödinger operators with $\delta$ and $\delta^{\prime}$ interactions on Lipschitz surfaces and chromatic numbers of associated partitions, Rev. Math. Phys. 26 (2014), 1450015, 43 pp.

[5] J. Behrndt, M. Langer, I. Lobanov, V. Lotoreichik and I. Yu. Popov, A remark on Schatten-von Neumann properties of resolvent differences of generalized Robin Laplacians on bounded domains, J. Math. Anal. Appl. 371 (2010), 750-758.

[6] J. Behrndt, M. Langer and V. Lotoreichik, Spectral estimates for resolvent differences of self-adjoint elliptic operators, Integral Equations Operator Theory, 77 (2013), 1-37.

[7] J. Behrndt, M. Langer and V. Lotoreichik, Schrödinger operators with $\delta$ and $\delta^{\prime}$ potentials supported on hypersurfaces, Ann. Henri Poincaré 4 (2013), 385-423.

[8] M. Sh. Birman, Perturbations of the continuous spectrum of a singular elliptic operator by varying the boundary and the boundary conditions, Vestnik Leningrad. Univ. 17 (1962), 22-55 (in Russian); translated in: Amer. Math. Soc. Transl. 225 (2008), $19-53$.

[9] M. Sh. Birman and M.Z. Solomjak, Asymptotic behavior of the spectrum of variational problems on solutions of elliptic equations, Sibirsk. Mat. Zh. 20 (1979), 3-22.

[10] M. Sh. Birman and M.Z. Solomjak, Asymptotic behavior of the spectrum of variational problems on solutions of elliptic equations in unbounded domains, Funktsional. Anal. i Prilozhen. 14 (1980), 27-35 (Russian); translated in: Funct. Anal. Appl. 14 (1981), 267-274.

[11] L. Boutet de Monvel, Boundary problems for pseudo-differential operators, Acta Math. 126 (1971), 11-51.

[12] J.F. Brasche, Upper bounds for Neumann-Schatten norms, Potential Anal. 14 (2001), 175-205.

[13] J. F. Brasche, P. Exner, Yu. A. Kuperin and P. Šeba, Schrödinger operators with singular interactions, J. Math. Anal. Appl. 184 (1994), 112-139.

[14] R. Brummelhuis and P. Duclos, Effective Hamiltonians for atoms in very strong magnetic fields, J. Math. Phys. 47 (2006), 032103, 41 pp.

[15] G. Carron, Déterminant relatif et la fonction Xi, Amer. J. Math. 124 (2002), 307-352.

[16] P. Deift and B. Simon, On the decoupling of finite singularities from the question of asymptotic completeness in two body quantum systems, J. Funct. Anal. 23 (1976), 218- 238

[17] V. Duchêne and N. Raymond, Spectral asymptotics of a broken delta interaction, J. Phys. A 47 (2014), 155203, 19 pp.

[18] P. Exner, Leaky quantum graphs: a review, in: Analysis on graphs and its applications. Selected papers based on the Isaac Newton Institute for Mathematical Sciences programme, Cambridge, UK, 2007. Proc. Symp. Pure Math. 77 (2008), 523-564.

[19] P. Exner, E. M. Harrell and M. Loss, Inequalities for means of chords, with application to isoperimetric problems, Lett. Math. Phys. 75 (2006), 225-233.

[20] P. Exner and I. Ichinose, Geometrically induced spectrum in curved leaky wires, J. Phys. A 34 (2001), 1439-1450.

[21] P. Exner and M. Jex, Spectral asymptotics of a strong $\delta^{\prime}$ interaction on a planar loop, J. Phys. A 46 (2013), 345201, 12 pp.

[22] P. Exner and S. Kondej, Bound states due to a strong $\delta$-interaction supported by a curved surface, J. Phys. A 36 (2003), 443-457. 
[23] P. Exner and K. Pankrashkin, Strong coupling asymptotics for a singular Schrödinger operator with an interaction supported by an open arc, Comm. Partial Differential Equations 39 (2014), 193-212.

[24] A. Figotin and P. Kuchment, Band-gap structure of spectra of periodic dielectric and acoustic media. II. Two-dimensional photonic crystals, SIAM J. Appl. Math. 56 (1996), 1561-1620.

[25] M. L. Gorbachuk and V. A. Kutovoŭ, Resolvent comparability of some boundary value problems, Funktsional. Anal. i Prilozhen. 16 (1982), 52-53.

[26] G. Grubb, Properties of normal boundary problems for elliptic even-order systems. Ann. Scuola Norm. Sup. Pisa Cl. Sci. (4) 1 (1974), 1-61.

[27] G. Grubb, Remainder estimates for eigenvalues and kernels of pseudodifferential elliptic systems, Math. Scand. 43 (1978), 275-307 (1979).

[28] G. Grubb, Singular Green operators and their spectral asymptotics, Duke Math. J. 51 (1984), 477-528.

[29] G. Grubb, Remarks on trace estimates for exterior boundary problems, Comm. Partial Differential Equations 9 (1984), 231-270.

[30] G. Grubb, Functional Calculus of Pseudodifferential Boundary Problems. Second edition. Birkhäuser, Boston, 1996.

[31] G. Grubb, Distributions and Operators. Graduate Texts in Mathematics, vol. 252. Springer, New York, 2009.

[32] G. Grubb, Perturbation of essential spectra of exterior elliptic problems, Appl. Anal. 90 (2011), 103-123.

[33] G. Grubb, Spectral asymptotics for Robin problems with a discontinuous coefficient, J. Spectr. Theory 1 (2011), 155-177.

[34] G. Grubb, The mixed boundary value problem, Krein resolvent formulas and spectral asymptotic estimates, J. Math. Anal. Appl. 382 (2011), 339-363.

[35] G. Grubb, Extension theory for elliptic partial differential operators with pseudodifferential methods, in: Operator Methods for Boundary Value Problems, London Math. Soc. Lecture Note Series, vol. 404 (2012), 221-258.

[36] G. Grubb, Spectral asymptotics for nonsmooth singular Green operators, Comm. Partial Differential Equations 39 (2014), 530-573.

[37] L. Hörmander, The spectral function of an elliptic operator, Acta Math. 121 (1968), 193-218.

[38] L. Hörmander, Fourier integral operators. I. Acta Math. 127 (1971), 79-183.

[39] S. Kondej and D. Krejčiřík, Spectral analysis of a quantum system with a double line singular interaction, Publ. Res. Inst. Math. Sci. 49 (2013), 831-859.

[40] S. Kondej and I. Veselić, Lower bounds on the lowest spectral gap of singular potential Hamiltonians, Ann. Henri Poincaré 8 (2007), 109-134.

[41] J. Lions and E. Magenes, Problèmes aux limites non homogénes et applications. Vol. 1, Travaux et Recherches Mathématiques, Dunod, Paris, 1968 (French); English translation: Non-Homogeneous Boundary Value Problems and Applications I, SpringerVerlag, Berlin-Heidelberg-New York, 1972.

[42] V. Lotoreichik and J. Rohleder, Schatten-von Neumann estimates for resolvent differences of Robin Laplacians on half-space, Oper. Theory Adv. Appl. 221 (2012), 471-486.

[43] M. M. Malamud, Spectral theory of elliptic operators in exterior domains, Russ. J. Math. Phys. 17 (2010), 96-125.

[44] I. Yu. Popov, The extension theory, domains with semitransparent surface and the model of quantum dot, Proc. Roy. Soc. London Ser. A 452 (1996), 1505-1515.

[45] A. Ya. Povzner, On the expansion of arbitrary functions in characteristic functions of the operator $\Delta u+c u$ (Russian), Mat. Sbornik N.S. 32 (1953), 109-156.

[46] S. Rempel and B.-W. Schulze, Parametrices and boundary symbolic calculus for elliptic boundary problems without the transmission property, Math. Nachr. 105 (1982), 45-149.

[47] R. Seeley, Complex powers of an elliptic operator, Amer. Math. Soc. Proc. Symp. Pure Math. 10 (1967), 288-307. 
[48] J. Shabani, Some properties of the Hamiltonian describing a finite number of $\delta^{\prime}$ interactions with support on concentric spheres, Nuovo Cimento B 101 (1988), 429437.

[49] T. A. Suslina and R. G. Shterenberg, Absolute continuity of the spectrum of the Schrödinger operator with the potential concentrated on a periodic system of hypersurfaces, Algebra i Analiz 13 (2001), 197-240 (in Russian); translated in: St. Petersburg Math. J. 13 (2002), 859-891.

[50] M. E. Taylor, Pseudodifferential Operators. Princeton Mathematical Series, vol. 34. Princeton University Press, Princeton, N.J., 1981.

Technische Universität Graz, Institut für Numerische Mathematik, SteyrERgasse 30, 8010 Graz, Austria

E-mail address: behrndt@tugraz.at

Department of Mathematical Sciences, Copenhagen University, UniversitetsParken 5, DK-2100, Copenhagen, Denmark

E-mail address: grubb@math.ku.dk

Department of Mathematics and Statistics, University of Strathclyde, 26 Richmond Street, Glasgow G1 1XH, United Kingdom

E-mail address: m.langer@strath.ac.uk

Technische Universität Graz, Institut für Numerische Mathematik, SteyrERgasse 30, 8010 Graz, Austria

E-mail address: lotoreichik@math.tugraz.at 